\title{
PRIMEROS RECONOCIMIENTOS SOBRE EL ESTADO DE CONSERVACIÓN DE PINTURAS RUPESTRES EN EL SECTOR DEL ALTO LOA, REGIÓN DE ANTOFAGASTA, CHILE
}

\section{INITIAL SURVEY OF THE STATE OF CONSERVATION OF ROCK PAINTINGS IN THE ALTO LOA AREA, ANTOFAGASTA REGION, CHILE}

\section{Paz Casanova*}

Se llevó a cabo el primer reconocimiento general del estado de conservación de las pinturas rupestres emplazadas en el sector del Alto Loa, Región de Antofagasta. Se evaluó la integridad física de 88 sitios arqueológicos, considerando la valoración y el uso de estos recursos culturales. Se estudió la relación entre las alteraciones registradas y los procesos involucrados en la ejecución de las manifestaciones. Los resultados indican la estabilidad de los sustratos y las pinturas, sin embargo, se identificó como punto vulnerable la pérdida de material causada por sales solubles y problemas de adhesión y cohesión de las mezclas pigmentarias. Se concluye que estos cambios derivan de factores intrínsecos de producción, los cuales diferencian la estabilidad de las pictografías rojas y el resto de los tonos registrados. La localización poco accesible de los soportes es un aspecto favorable en la conservación de los sitios, los cuales se encuentran mínimamente intervenidos por el ser humano.

Palabras clave: arte rupestre, conservación, procesos productivos, factores de alteración, indicadores de alteración

The paper describes the first general survey of the state of conservation of rock paintings located in the Alto Loa area, in Chile's Region II. The physical integrity of 88 archaeological sites was evaluated, taking into account the value and use of these cultural resources. The links between the alterations recorded and the processes involved in making the works was also studied. The results establish the stability of the substrata and paintings, but identify as a weak point the loss of material caused by soluble salts and problems with adhesion and cohesion of the pigments. The paper concludes that these changes result from factors intrinsic to production, which distinguish the red pictographs from those of other colors. The relative inaccessibility of the sites, which are minimally affected by humans, is favorable to their conservation.

Key words: rock art, conservation, production processes, alteration factors, alteration indicators

\section{INTRODUCCIÓN}

El desierto de Atacama es probablemente uno de los repositorios más importantes de pinturas rupestres en el norte de Chile y a pesar de los avances arqueológicos en la investigación de ellas, los estudios de conservación son inexistentes. En el presente artículo se da a conocer un balance preliminar del estado de conservación de las pinturas rupestres localizadas en el sector del Alto Loa, Región de Antofagasta. ${ }^{1}$ En el contexto de esta investigación, se identifican dos problemáticas relativas a este tema. La primera, se relaciona con la ausencia de un registro sistemático que dé cuenta de las condiciones de preservación de las manifestaciones rupestres. La segunda, se centra en la posible relación entre las alteraciones identificadas y los procesos involucrados en la producción de estas representaciones (Aschero 1988).

El sector del Alto Loa se reconoce como un importante patrimonio natural y cultural de la región atacameña. Su relevancia como referente identitario local, unido a su valor como fuente documental, ha promovido el estudio de la zona desde diferentes disciplinas. Puntualmente, las investigaciones sobre arte rupestre analizan el rol que cumplen estas manifestaciones, su significado (simbólico, funcional), su cronología y/o afiliaciones culturales y sus estilos (Berenguer et al. 1985; Berenguer \& Martínez 1986; Berenguer 1995, 1996, 1999, 2002, 2004a, 2004b;

* Paz Casanova, Centro de Investigaciones del Hombre en el Desierto, Universidad de Tarapacá, Av. General Velásquez 1320 A3 202, Arica, email: pazcasanov@gmail.com 
Cáceres \& Berenguer 1996; Horta 1996, 2000, 2001; Sepúlveda 2004; Vilches 2005).

Sin embargo, a pesar de los valores que desde diferentes esferas (Stanley Price 1995) son reconocidos en estos recursos, hasta la fecha no existe un diagnóstico orientado a conocer de forma sistemática su estado de conservación. ${ }^{2}$ Este hecho se ve agravado por cuanto en la zona existe un incipiente desarrollo de actividades de turismo informal, iniciativas que carecen de absoluto control y monitoreo. Esta situación, que puede incrementarse en el tiempo, dista de las recomendaciones y los principios establecidos en diferentes documentos redactados por organizaciones internacionales que velan por el desarrollo de un turismo cultural sustentable (OMT 1999; ICOMOs 1999; UNESCO 2006, entre otras).

Tomando en cuenta estos hechos, se aprovecha el contexto del proyecto FONDECYT 1070083 para efectuar un primer reconocimiento general del estado de conservación de las pinturas rupestres localizadas en el sector. Estableciendo el carácter global de la evaluación, se considera que esta iniciativa es un aporte a la identificación preliminar de indicadores y factores de alteración presentes en los recursos (Brunet et al. 1985; Martín 1990; Wainwright 1995; Bednarik 1995, 2003; Brunet \& Vouvé 1996; Watchman 2002; Morwood 2003). Desde la óptica de la conservación preventiva (Brunet 2006), este sería un primer paso en el intento de comprender los mecanismos de alteración que afectan a las manifestaciones. Adicionalmente, la generación de documentación escrita y visual crea un referente para monitorear y evaluar los cambios que puedan ocurrir en un futuro (Marymor 2001). Por último, los resultados obtenidos en esta etapa de evaluación permiten reflexionar sobre la necesidad de nuevas acciones de conservación, la pertinencia o no de intervenciones de tipo directa (Brunet et al. 1990) y las consecuencias que podría significar el uso de los sitios arqueológicos como recurso turístico.

A través de esta evaluación de conservación también se pretende aportar en la problemática netamente arqueológica, pues se intenta

descubrir hasta qué punto los pintores de distintas épocas prehispánicas de la región atacameña y áreas vecinas compartieron no solo el acceso a medios de producción pictóricos, sino también al conocimiento requerido para la ejecución y construcción visual de sus obras (Gallardo 2007 Ms: 5).

Siguiendo la hipótesis de que "la pintura rupestre es considerada como un conjunto de elecciones técnicas, tanto a nivel de la producción de la obra como de los dispositivos gráficos, que finalmente dan como resultado este tipo particular de artefactos visuales" (Gallardo 2007 Ms: 6-7), consideramos que, desde el punto de vista de la preservación de los recursos, estas elecciones también inciden, directa o indirectamente, en su preservación a lo largo del tiempo. Esta particularidad, que involucra los aspectos materiales y formales de las obras (Aschero 1988), es fundamental si se pretende indagar en la génesis de las alteraciones observadas.

Las características de los soportes seleccionados en términos materiales y morfológicos (Aschero 1988) son variables que forman parte del ambiente litológico de los sitios rupestres (Rocchietti 2003). Por lo tanto, desde este punto de vista, la particularidad de los recursos se traduce en la interacción de estos con determinados factores de alteración, tanto externos (atmosféricos y antrópicos) como internos (propiedades geológicas de las rocas) (Wainwright 1995; Bednarik 1995, 2003; Brunet \& Vouvé 1996; Morwood 2003, entre otros). Similar relación se puede establecer si se consideran los diferentes aspectos involucrados en la ejecución de las representaciones. Las características materiales y formales de las manifestaciones significan ciertas propiedades intrínsecas de los recursos, las cuales pueden sufrir modificaciones físicas y/o químicas a través del tiempo.

Esperando que en un futuro sea posible efectuar un diagnóstico más exhaustivo de las pictografías del sector, por el momento se propone como segundo objetivo un avance en la identificación de vínculos entre los procesos de manufactura de las pinturas del sector (selección de los soportes y ejecución de las representaciones) y los factores e indicadores de alteración registrados en estos recursos.

En definitiva, a través de este reconocimiento se espera, prioritariamente, evaluar y documentar el estado de conservación de los recursos y, posteriormente, establecer en qué medida las alteraciones registradas se vinculan a los procesos involucrados en su producción.

\section{METODOLOGÍA DE DIAGNÓSTICO}

\section{El área de estudio}

El río Loa, ubicado en la Región de Antofagasta, nace en la falda norte del volcán Miño en Ojos del Miño (3.900 msnm) y desemboca en el océano Pacífico a la altura de Caleta Huelén. Con una longitud de $440 \mathrm{~km}$ y conformando varios lechos, atraviesa las unidades geomorfológicas de la Región.

Su régimen hídrico es de carácter pluvial, ya que sus crecidas obedecen a intensas lluvias de verano, caídas en la alta cordillera, producto del denominado "invierno altiplánico". Los montos de las precipitaciones se incrementan conforme a la altura y disminuyen en sentido norte-sur. Esta gradiente pluviométrica relacionada 
con la altitud permite el desarrollo de vegetación zonal constituida por arbustos xerófilos, además de una vegetación de carácter azonal en el piso del valle formada por gramíneas de altura y colas de zorro (Berenguer 2004a). En este sector se presentan los climas desierto normal y desértico marginal de altura (DGAC 2009).

El área de estudio involucrada en el proyecto arqueológico comprende la zona denominada Alto Loa, la cual hace referencia exclusivamente al brazo superior de esta cuenca hidrográfica (Berenguer et al. 2005). ${ }^{3}$ Este tramo, que abarca desde su nacimiento hasta la confluencia con el río Salado, presenta un curso norte-sur, el cual recorre casi $150 \mathrm{~km}$ de escarpadas terrazas fluviales.

El área específica donde se realizó el estudio de conservación corresponde al sector Santa Bárbara, tramo intermedio del brazo cordillerano del Loa (véase Berenguer 2004b). En este sector, el curso del río recorre un cañón formado por paredes verticales de roca volcánica, caracterizadas por tobas cineríticas e ignimbritas soldadas de carácter riolítico (Niemeyer 1967).

Con respecto a las pinturas que se encuentran en esta zona, la literatura especializada describe como principales estilos los denominados Taira, La Isla, Milla y Santa Bárbara (véase Berenguer 1999, 2002, 2004a, 2004b). ${ }^{4}$ Sobre la información cronológica de los sitios se estiman ocupaciones desde 795-390 AC (datación efectuada en Taira sitio tipo) hasta 1450 DC, es decir, desde el Período Formativo al Período Intermedio Tardío (Cáceres \& Berenguer 1996; Horta 1996; Berenguer 2004a).

\section{Selección de los sitios y paneles evaluados}

Luego de que el equipo arqueológico definió las unidades de análisis (sitios, paneles, motivos), se delimitó la muestra a evaluar. Las variables relevantes de selección de los sitios fueron: a) la valoración del sitio, es decir, su importancia intrínseca desde el punto de vista científico, estético, educativo, etcétera; b) la existencia de al menos un punto de observación que propicie su evaluación; c) la unicidad y/o singularidad del sitio y/o representaciones; d) el grado de dificultad de acceso al lugar, y e) la actual incorporación de los emplazamientos a circuitos turísticos y/o visitas informales. Se seleccionaron sitios emplazados en ambos lados de la quebrada.

En cada uno de los sitios seleccionados se registró el total de los paneles presentes. Esta metodología difiere de la desarrollada por el equipo de arqueólogos del proyecto, quienes registraron solo los que presentaban motivos con un mínimo grado de definición formal, excluyendo los completamente alterados.

Considerando todos los atributos señalados, se recorrió un área que abarcó aproximadamente 11 km dirección norte-sur, comenzando $400 \mathrm{~m}$ al norte del alero Taira y finalizando en el alero Santa Bárbara (fig. 1). Durante diez días, un fotógrafo y dos conservadores registraron 88 sitios, lo cual significó un total de 210 paneles, universo total de la muestra.

\section{Reconocimiento general del estado de conservación de las pinturas rupestres}

Por este procedimiento se entiende la identificación y el registro de los principales factores e indicadores de alteración, observables a simple vista, que signifiquen un actual o potencial cambio en la integridad física de las pinturas (capa pictórica y soporte). ${ }^{5}$ Con el fin de cumplir este primer objetivo, los procedimientos utilizados se basan en propuestas de otros proyectos -nacionales e internacionales- ante problemáticas similares en términos ambientales y/o de gestión de los recursos (Soleilhavoup 1985; Bellelli et al. 1999; Ávalos \& Ladrón de Guevara 2000; Podestá et al. 2000; Rolandi et al. 2002; Ladrón de Guevara 2004; Bellelli et al. 2005, entre otros).

A través de una herramienta cualitativa de registro se relevaron in situ los principales indicadores y factores de alteración apreciables a simple vista, tanto en los sitios arqueológicos como en los soportes rocosos y las capas pictóricas. Estos valores se proponen a partir de variables descritas por Brunet y colaboradores (1985), Martín (1990), Brunet (1995), Wainwright (1995), Bednarik (1995, 2003), Brunet y Vouvé (1996); Meléndez y Fuster (1998), Watchman (2002), Morwood (2003) y Alonso y colaboradores (2006). Entre los indicadores de alteración se consignaron los cambios en el aspecto externo de los soportes (pátinas, costras, depósitos superficiales [sedimentos, concreciones, sales, microorganismos, excrementos, manchas, nido de insectos], oxidación); la pérdida de los soportes (cavidades, erosión, decohesión); las rupturas y disyunciones de estos (grietas, fisuras, fragmentación, deplacamiento, exfoliación); la pérdida de configuración de las representaciones (desprendimiento, fisuras, pulverulencia, riesgo de desprendimiento); la pérdida de intensidad cromática de estas (lavado, depósitos superficiales); las variaciones cromáticas de saturación y/o valor y las intervenciones antrópicas (grafiti, repaso, incisiones, ahumado, otros). Entre los factores de alteración se relevaron los de origen extrínseco (agentes atmosféricos y antrópicos) e intrínsecos (propiedades de la mezcla pigmentaria y soportes).

Para efectuar una caracterización general de los sitios y las pinturas rupestres se consideraron diversos atributos propuestos por Gradín (1978), Hernández Llosas (1985) y Rocchietti (2003). A partir del esquema 


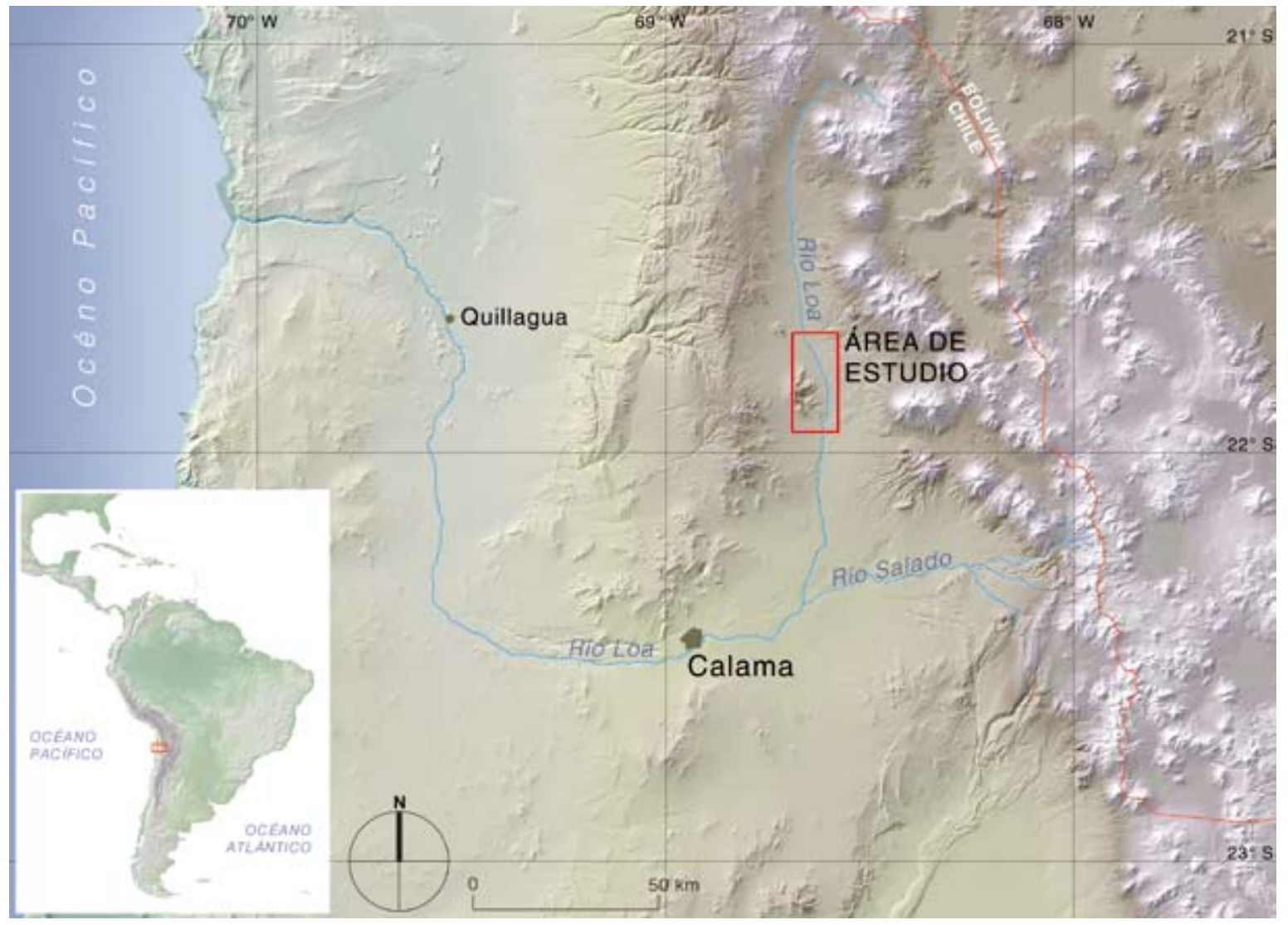

Figura 1. Mapa de área de estudio de pinturas rupestres, sector del Alto Loa.

Figure 1. Map of the area under study, Alto Loa sector.

Tabla 1. Síntesis de los principales indicadores y factores de alteración registrados en 88 sitios con pictografías en el sector del Alto Loa.

Table 1. Summary of main alteration indicators and factors recorded at 88 pictograph sites in the Alto Loa sector.

\section{Indicador de alteración \\ Pérdida de configuración de las} representaciones.

Pérdida de configuración de las representaciones.

Pérdida de configuración de las representaciones.

Pérdida de intensidad cromática de las figuras.

Pérdida de intensidad cromática de Geofísico/viento/antrópico. las figuras.

Pérdida de intensidad cromática de Antrópico. las figuras.

\section{Factor/agente}

Factores intrínsecos no determinados.

Factores intrínsecos no determinados.

Hidrológico/geofísico/viento/ sales solubles.

Hidrológico/agua lluvia.

Manifestación
$\begin{aligned} & \text { Pérdida de adhesión de la capa pictórica } \\ & \text { (pictografías blancas). }\end{aligned}$

Pérdida de cohesión de la capa pictórica (pictografías verdes y negras).

Subflorescencia, exfoliación de los sustratos y pérdida de capa pictórica.

Lavado de las figuras por escurrimiento de aguas lluvia sobre las superficies.

Abrasión de partículas transportadas por el viento.

Depósitos superficiales.

Incisión de pictografías localizadas dentro de aleros. 
de producción de las representaciones planteado por Aschero (1988), se consignaron solo las variables posibles de determinar a nivel macroscópico: a) localización de los soportes, considerando principalmente las características topográficas de estos y su condición natural de reparo y exposición, y b) ejecución de las representaciones, distinguiendo los aspectos materiales (consistencia de la mezcla pigmentaria), técnicos (tratamiento de las superficies) y formales (identificación de los principales conjuntos tonales) presentes en cada panel (Munsell 2000). ${ }^{6}$ Esta labor se ejecutó al mismo tiempo por el grupo de arqueólogos y el de conservadores, comparando posteriormente los datos recabados.

\section{Sistematización y análisis de los datos recopilados in situ}

Los datos se sistematizaron a través de tablas de frecuencia porcentual, las que incluyen la distribución de los principales indicadores de alteración presentes en las pinturas y las variables involucradas en los procesos de manufactura de estas (soportes y ejecución de las representaciones)

A través de la construcción de tablas de contingencia, se buscó obtener una descripción cuantitativa de los indicadores de alteración y de las variables de producción. Posteriormente, se analizaron las relaciones entre las variables a) soportes/factores/indicadores de alteración y b) ejecución de las representaciones/ factores/indicadores de alteración.

Finalmente, se realizó una síntesis de los principales factores e indicadores de alteración jerarquizándolos según el grado de impacto que significan sobre la preservación de los recursos. Se consideraron las variables cualitativas de intensidad de la acción, su extensión (superficie que abarca), su prolongación en el tiempo (activo, cíclico, inactivo) y la posibilidad de reversión de la misma (Ávalos \& Ladrón de Guevara 2000).

\section{RESULTADOS}

Se entregan los resultados obtenidos a partir de la identificación de los principales indicadores de alteración (Gráfico 1) y el análisis de la relación entre soportes/ factores/indicadores de alteración y ejecución de las representaciones/factores/indicadores de alteración. Finalmente se presenta una síntesis de los indicadores de mayor impacto sobre la integridad física de las representaciones (Tabla 1).

\section{Soportes de las pinturas rupestres}

Se diferenció entre cinco categorías de sustratos utilizados como soporte de las pinturas rupestres (Gráfico 2). Esta clasificación obedece específicamente a la localización y

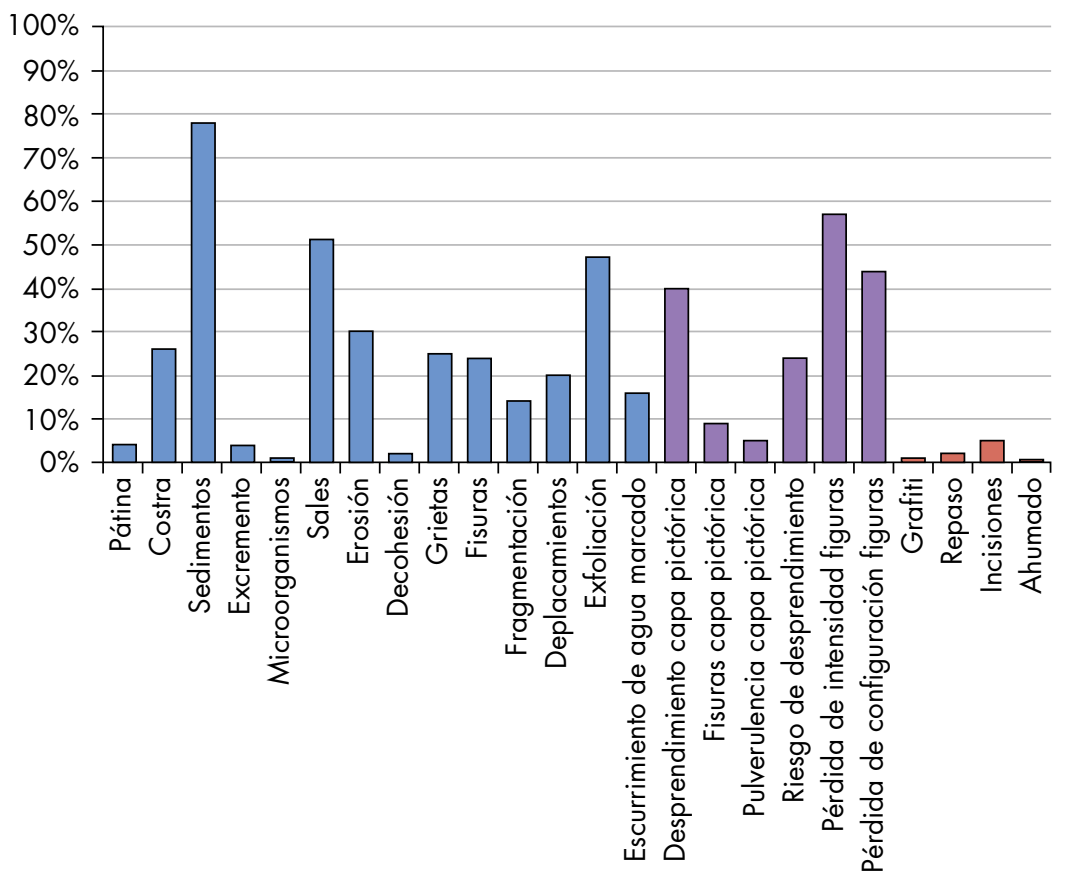

Gráfico 1. Principales indicadores de alteración registrados en 210 paneles con pinturas rupestres en el sector del Alto Loa. Graph 1. Main alteration indicators recorded on 210 rock art panels in the Alto Loa sector. 
el tipo morfológico que estos presentan a partir de diferentes procesos geodinámicos de formación (Wainwright 1995) y de posterior meteorización (Meléndez \& Fuster 1998). ${ }^{7}$ Esta condición conlleva, además, diferencias en términos de reparo, exposición y visibilidad de las representaciones.

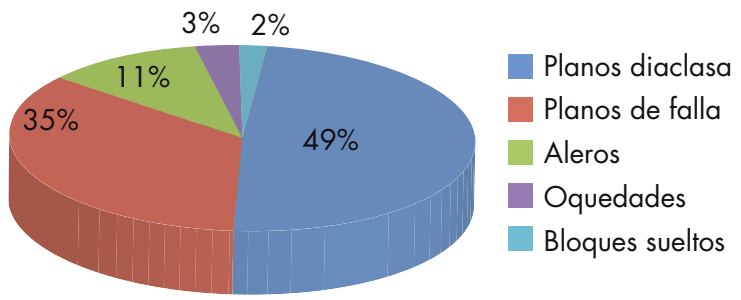

Gráfico 2. Distribución de frecuencia porcentual de la localización de los sustratos utilizados como soportes de pinturas rupestres, sector del Alto Loa.

Graph 2. Frequency distribution of substrates used as rock art supports, Alto Loa sector.

Un 98\% de los paneles registrados se localizan en ambos lados de la pared riolítica del cañón, sobre el talud de escombros de falda, a una altura de entre $30 \mathrm{y}$
$150 \mathrm{~m}$ sobre el río Loa (3.100 msnm promedio) (fig. 2). El 2\% restante se ubica en bloques sueltos sobre la pendiente. Entre los soportes más representativos en términos porcentuales se distinguen los derivados de procesos geodinámicos internos (tectónicos), los que se identifican como planos secundarios conformados por diaclasas y fallas en la roca.

Los planos conformados por diaclasas ( $n=104 ; 49 \%$ ), es decir, fracturas de la pared sin desplazamiento de la roca, conforman superficies generalmente irregulares, cuadrangulares y delimitadas por grietas de dirección vertical, horizontal u oblicua (fig. 3). Algunos de estos conjuntos se encuentran en sectores aterrazados que tienen entre dos a seis metros de profundidad, conformando espacios más protegidos y de escasa visibilidad desde el lecho del río. Las representaciones varían en su tamaño, registrándose motivos de entre diez centímetros hasta más de un metro de alto. En algunos casos se utilizan planos muy cercanos al piso y en otros a más de dos metros de altura.

Los planos de falla $(\mathrm{n}=73 ; 35 \%)$, resultado de la fractura y el desplazamiento de las rocas, dan forma a grandes superficies, generalmente de textura muy lisa, localizadas en la parte superior del cañón (fig. 4). Por

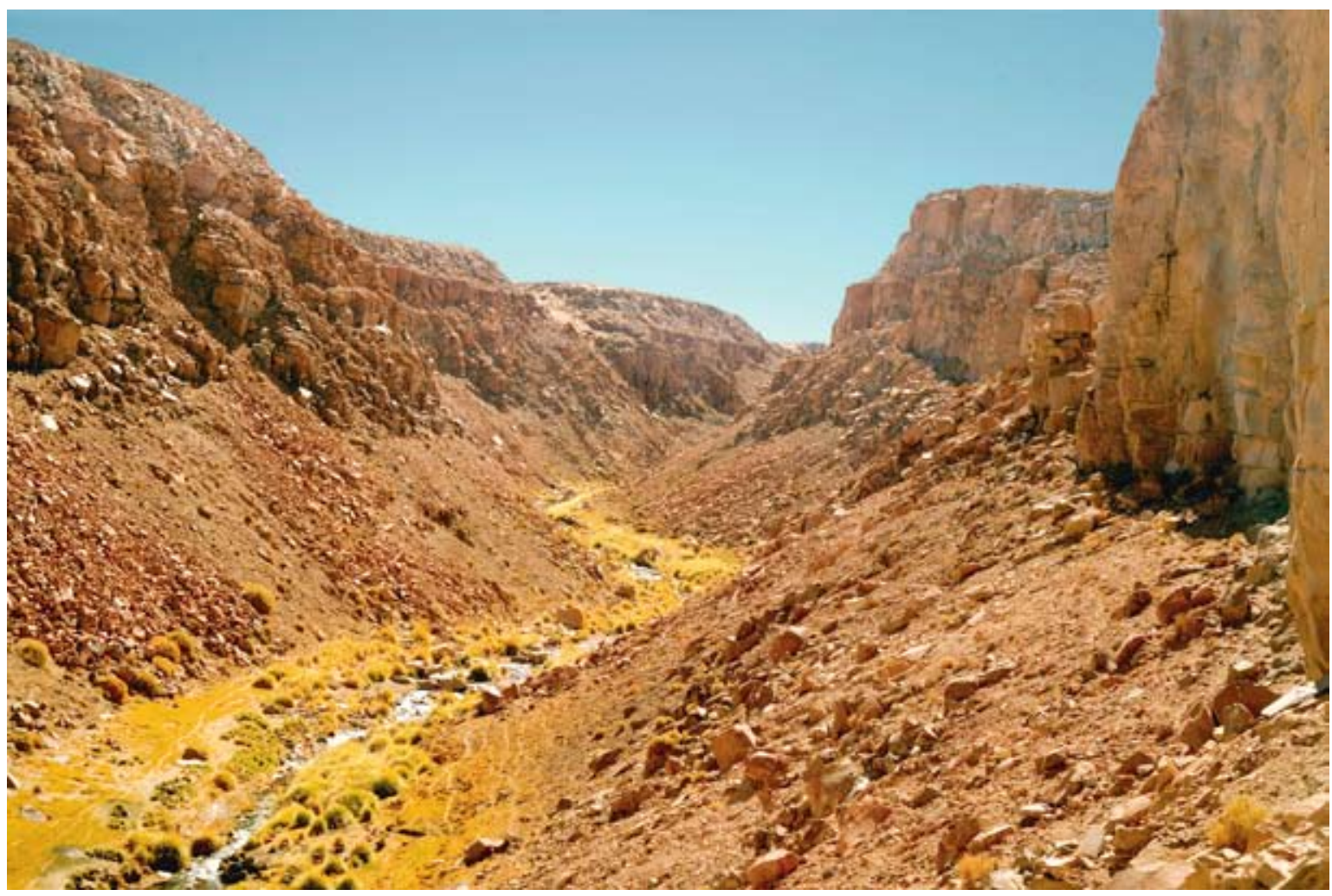

Figura 2. Vista panorámica del sector del Alto Loa. Las pictografías se localizan en la pared del cañón sobre el talud de escombros de falda (foto: I. Navarro, 2007).

Figure 2. View of the Alto Loa sector. The pictographs are located on the ravine wall above the lower screen slope (photo: I. Navarro, 2007). 


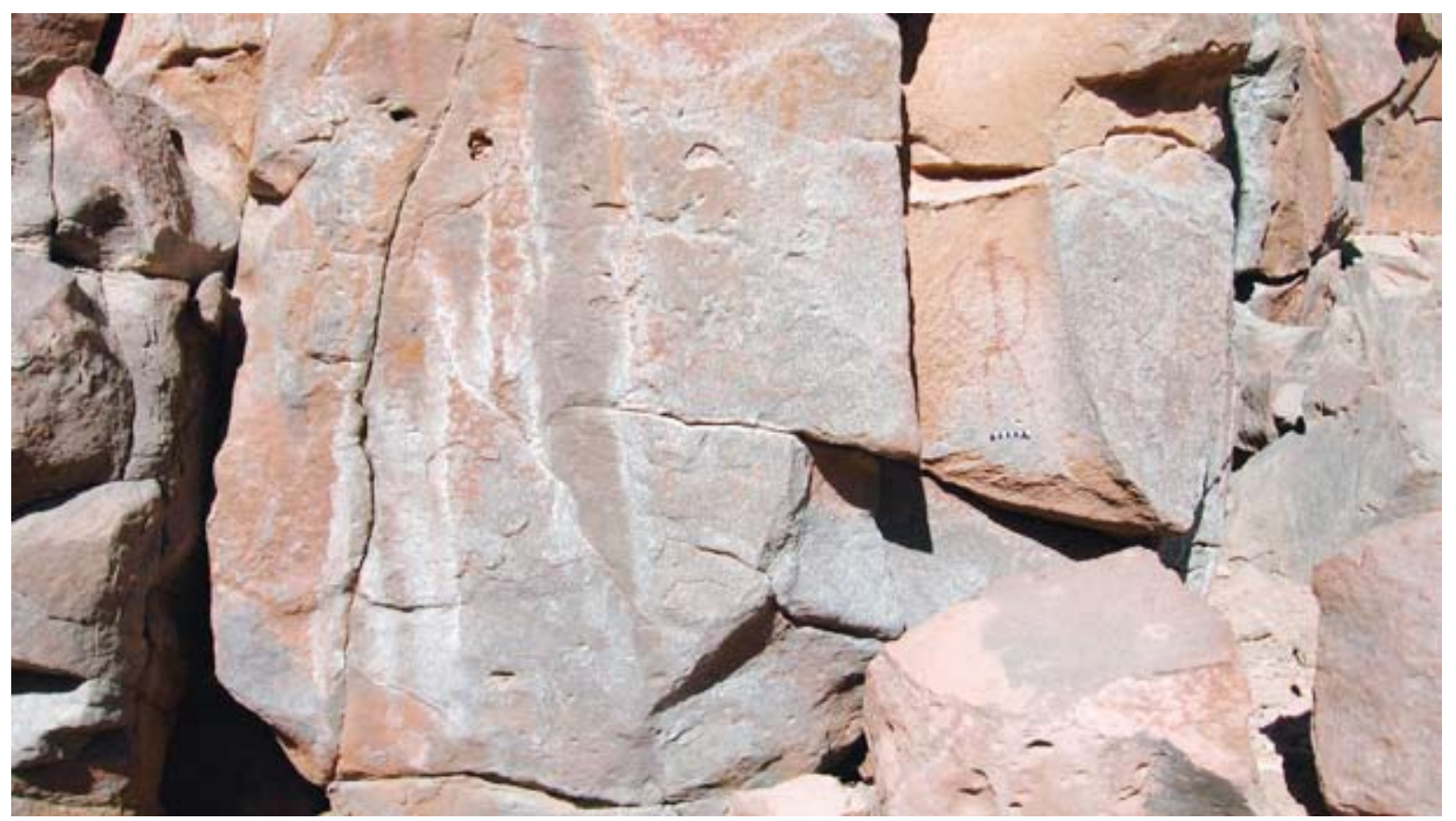

Figura 3. Planos asociados a diaclasas utilizados como soporte de pictografías, Alto Loa (foto: P. Casanova, 2007). Figure 3. Rock face used as a pictographic medium, Alto Loa (photo: P. Casanova, 2007).

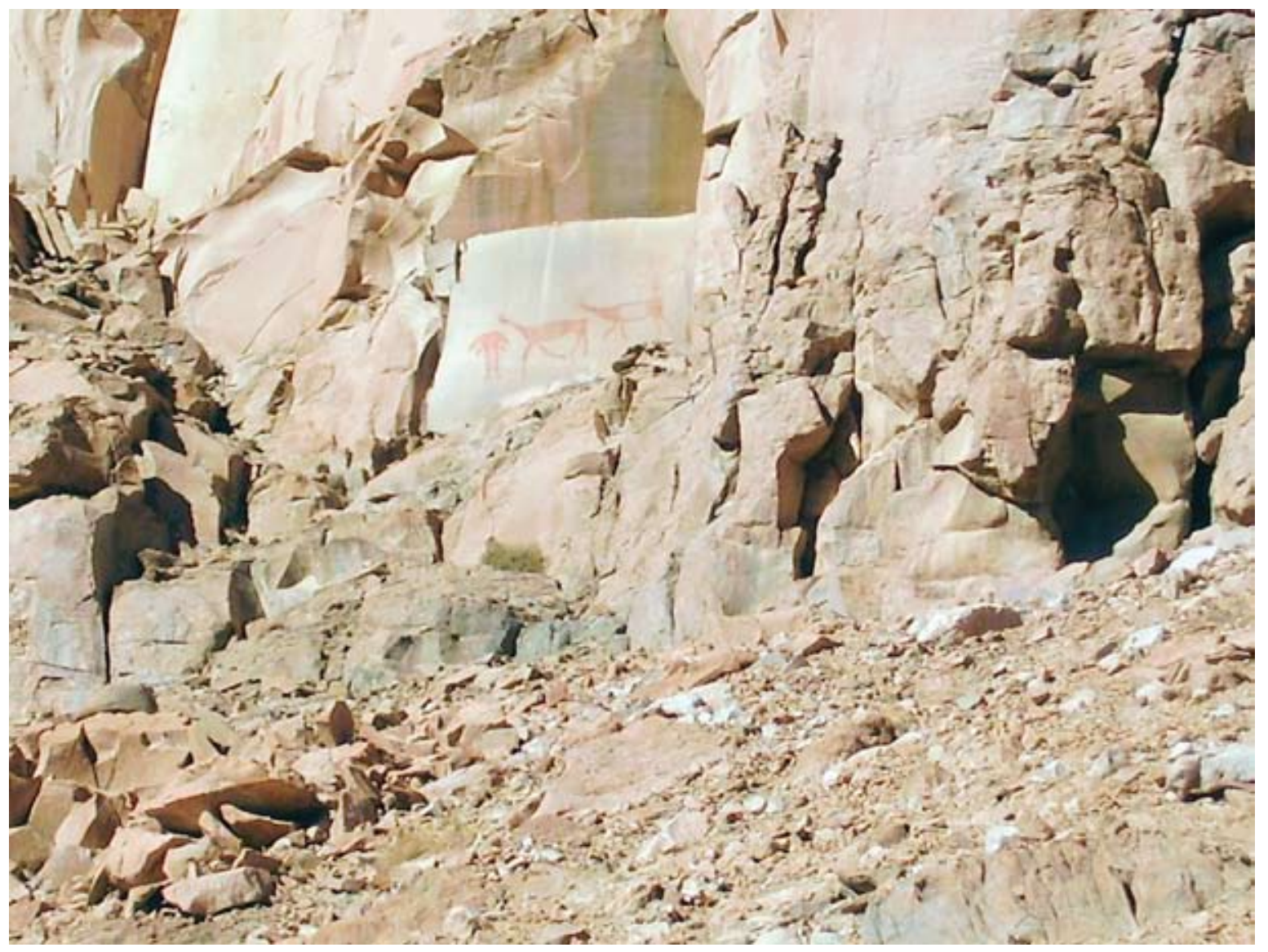

Figura 4. Planos de falla utilizados como soporte de pictografías, Alto Loa (foto: P. Casanova, 2007).

Figure 4. Fault plane used as a pictographic medium, Alto Loa (photo: P. Casanova, 2007). 
lo general, desde el sendero superior por donde se accede a los sitios, no es posible obtener un punto de observación adecuado para apreciar la globalidad de las representaciones, ya que algunas de ellas superan los dos metros de altura. En estos casos, mejor perspectiva se tiene desde el fondo del cañón.

Por otra parte, un $11 \%$ de los paneles se encuentran dentro de aleros naturales, mayoritariamente asociados a estructuras pircadas (fig. 5). Estos abrigos son producto de procesos de intemperismo que han afectado de forma diferencial las bases de las paredes rocosas provocando su socavamiento. Las pinturas realizadas en estos recintos se encuentran tanto en el interior como en el exterior de los muros.

Otro lugar de localización de las pinturas son las oquedades conformadas en la pared del cañón (fig. 6). Estos espacios vacíos formados por procesos de alteración sobre las superficies verticales de las rocas representan un $3 \%$ de los soportes.

Por último, la utilización de bloques sueltos sobre el talud se registra en un $2 \%$ (fig. 7).

\section{Relación entre soportes, factores e indicadores de alteración}

Las relaciones más relevantes entre los factores e indicadores de alteración y la localización de los soportes son: a) la pérdida de intensidad cromática de las figuras registradas en los planos de falla; b) sales y sedimentos superficiales localizados en planos de diaclasas, y c) las incisiones registradas dentro de los aleros. En cuanto a los factores que favorecen el desarrollo de estas alteraciones se determina que estos son principalmente de origen extrínseco y se identifican como hidrológicos, geofísicos y, en menor medida, antrópicos. Según la localización de los paneles se registró una variación en la intensidad y los mecanismos de acción de los agentes, observándose una distribución diferencial de los indicadores de alteración entre los cinco tipos de soportes (Gráfico 3).

En este sector, el agua de lluvia y la humedad son los principales agentes de alteración relacionados con el factor hidrológico. La lluvia, de presencia ocasional y de

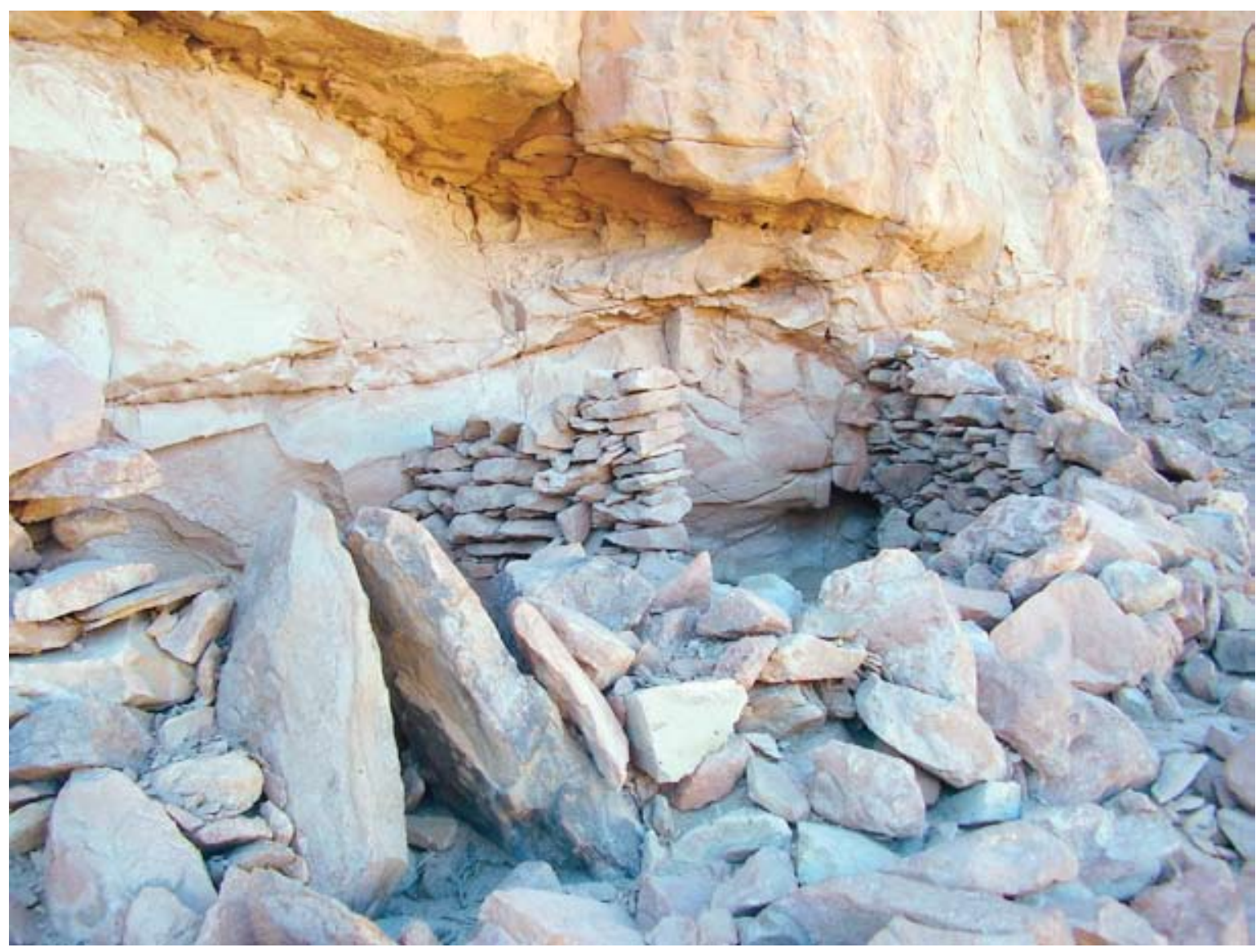

Figura 5. Pictografías localizadas en soportes al interior de alero, Alto Loa (foto: P. Casanova, 2007).

Figure 5. Rock shelter wall used for pictographs, Alto Loa (photo: P. Casanova, 2007). 


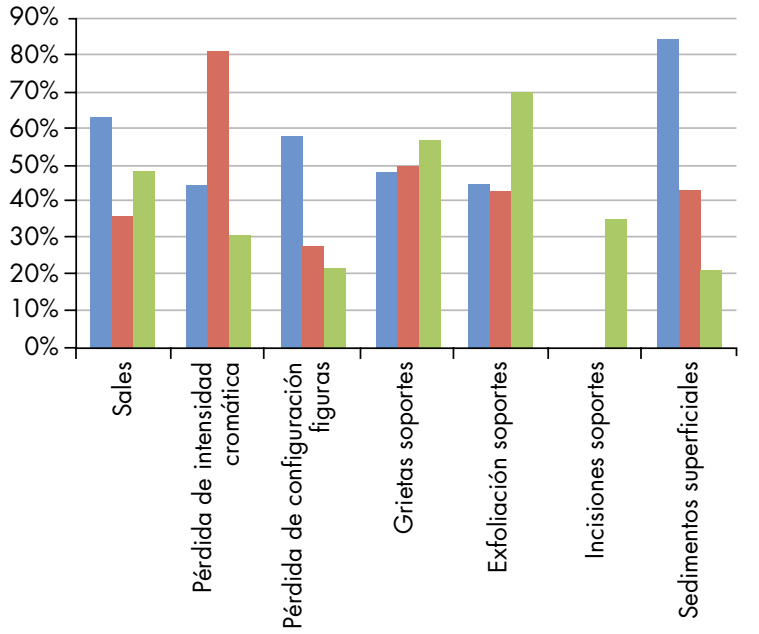

Planos diaclasa

Planos de falla

Aleros

Gráfico 3. Distribución de los principales indicadores de alteración según localización de los sustratos más utilizados como soportes de pinturas rupestres, sector del Alto Loa.

Graph 3. Distribution of main alteration indicators by location of substrates most often used for paintings, Alto Loa sector. intensidad variable, se encuentra asociada principalmente a precipitaciones estivales, entre enero y comienzos de marzo. Aunque los valores registrados en el embalse de Conchi $(2.932 \mathrm{msnm})$ presentan un promedio de solo $17,3 \mathrm{~mm}$ (Narbona 2009), en los planos de fallas este es un agente importante de alteración considerando que un $92 \%$ de estos presentan un nivel de exposición alto. ${ }^{8}$ Esta condición significa que no existen barreras físicas que impidan que el agua escurra directamente sobre las superficies. Adicionalmente, los suelos salinos de poca permeabilidad predominante en el alto de la quebrada presentan importantes niveles de escorrentía, flujos que también pueden descender directamente sobre las paredes del cañón. El agua de lluvia, que nunca es absolutamente pura, puede transportar compuestos degradantes de las rocas (Watchman 2002; Bednarik 2003) y también generar la acción abrasiva de las partículas en ella suspendidas. Finalmente, esta situación se manifiesta como una pérdida de la intensidad cromática de las figuras (fig. 8; Brunet et al. 1996). Esta alteración es significativa pues se registra en un $81 \%$ de los paneles

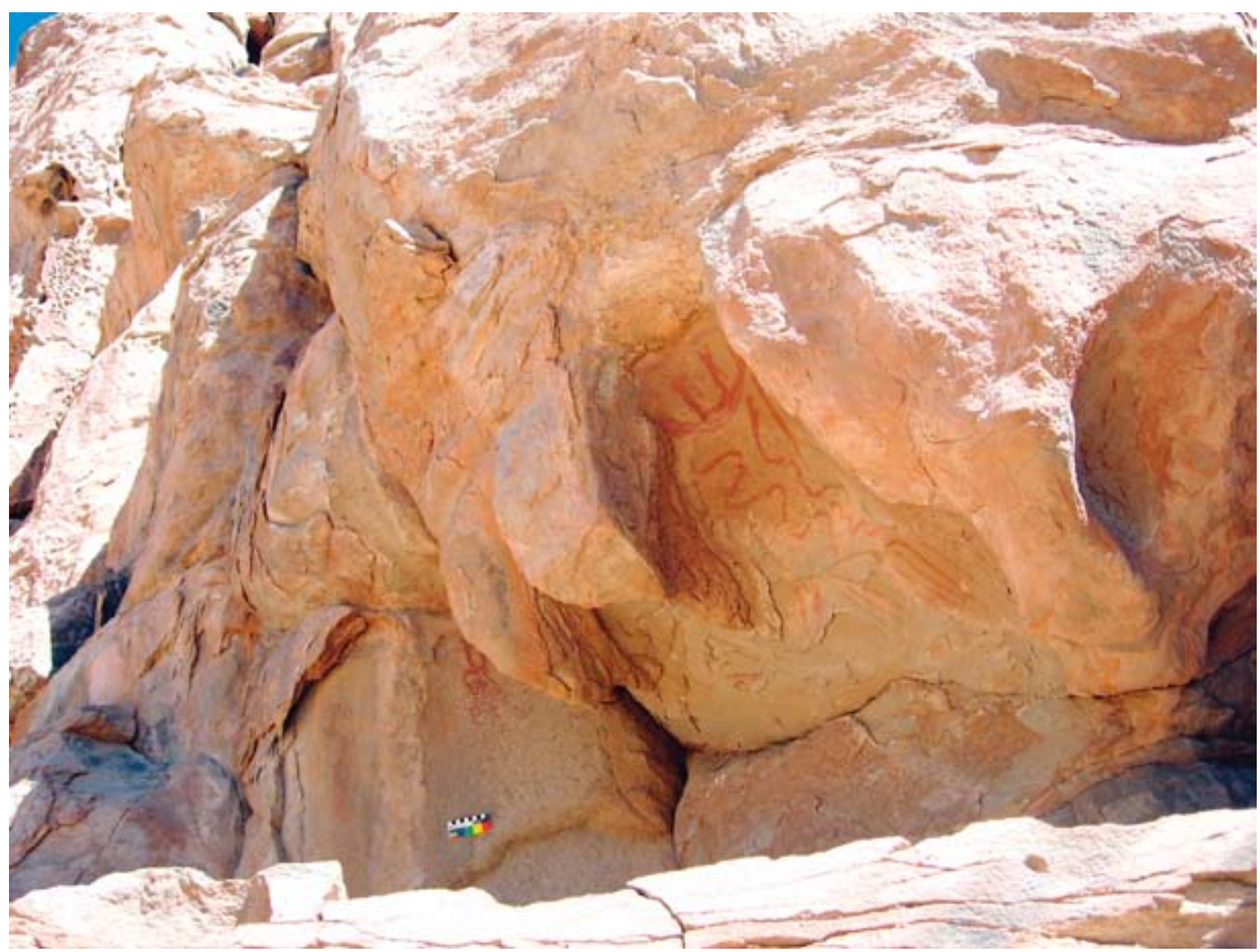

Figura 6. Sector Cueva de La Damiana. Pictografía sobre oquedad de pared, Alto Loa (foto: P. Casanova, 2007).

Figure 6. Cueva de La Damiana sector. Pictographs in the hollows of a rock wall, Alto Loa (photo: P. Casanova, 2007). 


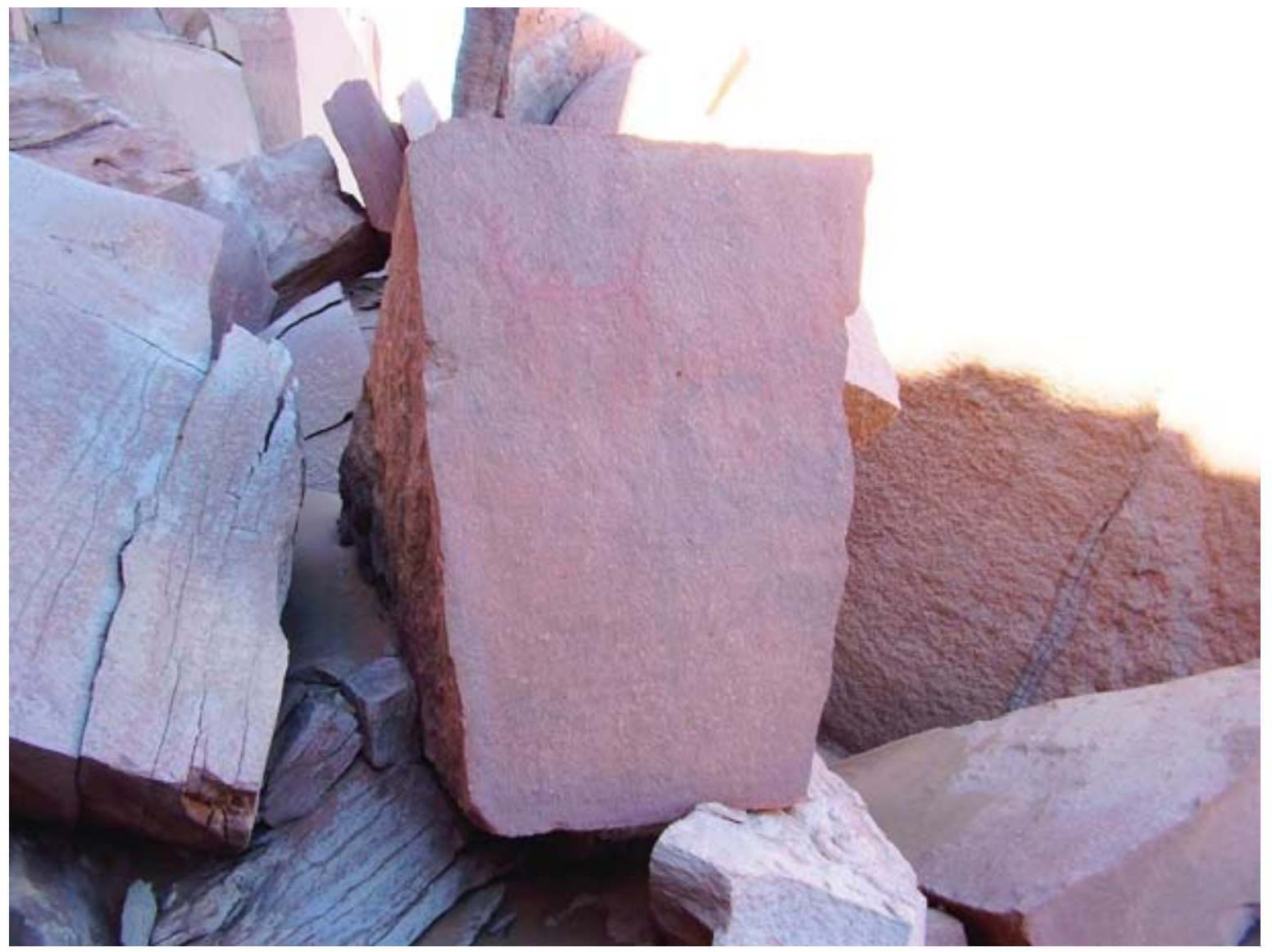

Figura 7. Sitio LS046. Pictografía sobre bloque suelto, Alto Loa (foto: P. Casanova, 2007).

Figure 7. Site LSO46. Pictograph on loose boulder, Alto Loa (photo: P. Casanova, 2007).

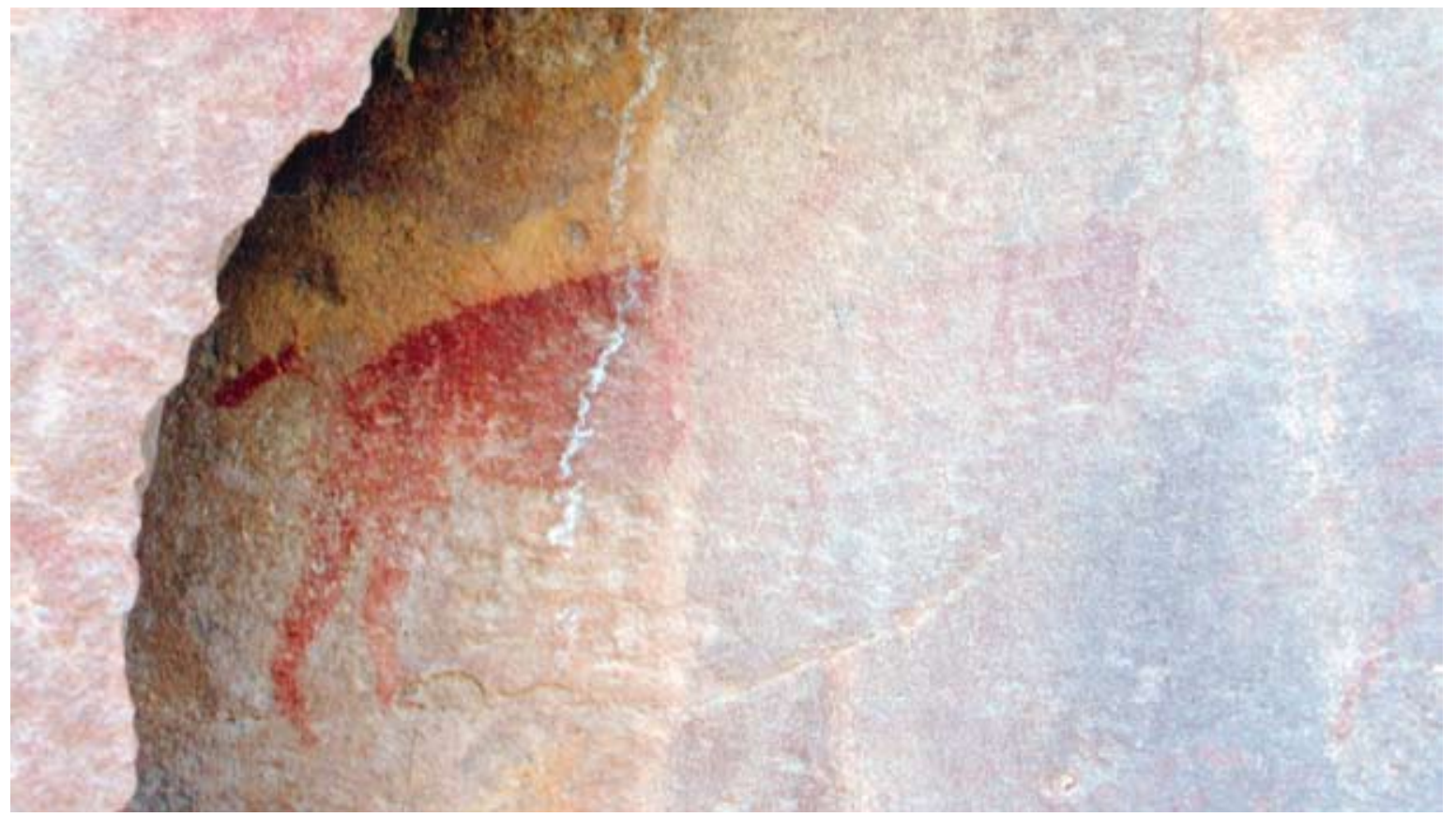

Figura 8. Pérdida de configuración formal de la pictografía producto de escurrimiento de agua, Alto Loa (foto: P. Casanova, 2007). Figure 8. Loss of pictograph form due to water runoff, Alto Loa (photo: P. Casanova, 2007). 
localizados en planos de falla, mientras que en los otros soportes no supera el $44 \%$.

Los planos asociados a diaclasas se encuentran en sectores donde existe cierto grado de reparo y, por tanto, el agua lluvia no cae directamente sobre las pinturas, sin registrarse un alto porcentaje de pinturas lavadas. Sin embargo, el agua lluvia igualmente se filtra por las grietas de los bloques, arrastrando compuestos que se encuentran acumulados en estas hendiduras y en la superficie de la roca. El agua facilita la movilización de las sales solubles sobre los sustratos, generándose en primera instancia una película blanquecina que altera las características cromáticas de las representaciones. Por otra parte, la humedad, considerada como un agente principal en la destrucción del arte rupestre (Bednarik 1995, 2003), al ser absorbida por la roca introduce la sal depositada sobre las superficies (subflorescencias). Ciclos de condensación intersticial y evaporación generan la cristalización de las sales, provocando la ampliación de fisuras, un abombamiento de las superficies, la exfoliación de los soportes y, finalmente, la pérdida de la configuración de las representaciones. Este nivel de alteración no es muy común, pero en los casos en que se manifiesta el estado de las pictografías es seriamente frágil (fig. 9). Se debe considerar que los agentes naturales responsables de estos procesos actúan de forma interdependiente. La presencia de sales higroscópicas y sedimentos sobre las superficies produce un aumento importante en la absorción de agua (Fort 1996), lo cual favorece a su vez estos mecanismos de alteración.

La acumulación de sedimentos sobre la roca se atribuye a la acción del viento, el cual es un agente natural relevante en el sector, que presenta grados de intensidad importantes, pero variables. Se constata que en ambos lados de la pared del cañón no existe vegetación alguna que actúe como barrera física ante su presencia. Como se señaló, los soportes pictóricos asociados a planos de diaclasas se encuentran preferentemente en sitios protegidos y aterrazados. En estos espacios, donde el área del suelo es mayor, el viento provoca un efecto de remolino, lo que lleva a la depositación de las partículas sobre las paredes, obliterando las pinturas (fig. 10). Adicionalmente, a través de procesos de acción eólica también se depositan sobre los soportes los productos residuales presentes en el talud. En las pinturas localizadas sobre planos de falla se registra un menor porcentaje de este tipo de alteración, sin embargo, la fase de erosión del material particulado provoca la abrasión de los soportes y, a largo plazo, su disgregación granular.

Con respecto a los factores antrópicos, se observaron excavaciones arqueológicas en varios de los sitios aterrazados, actividad que también ha aportado en la

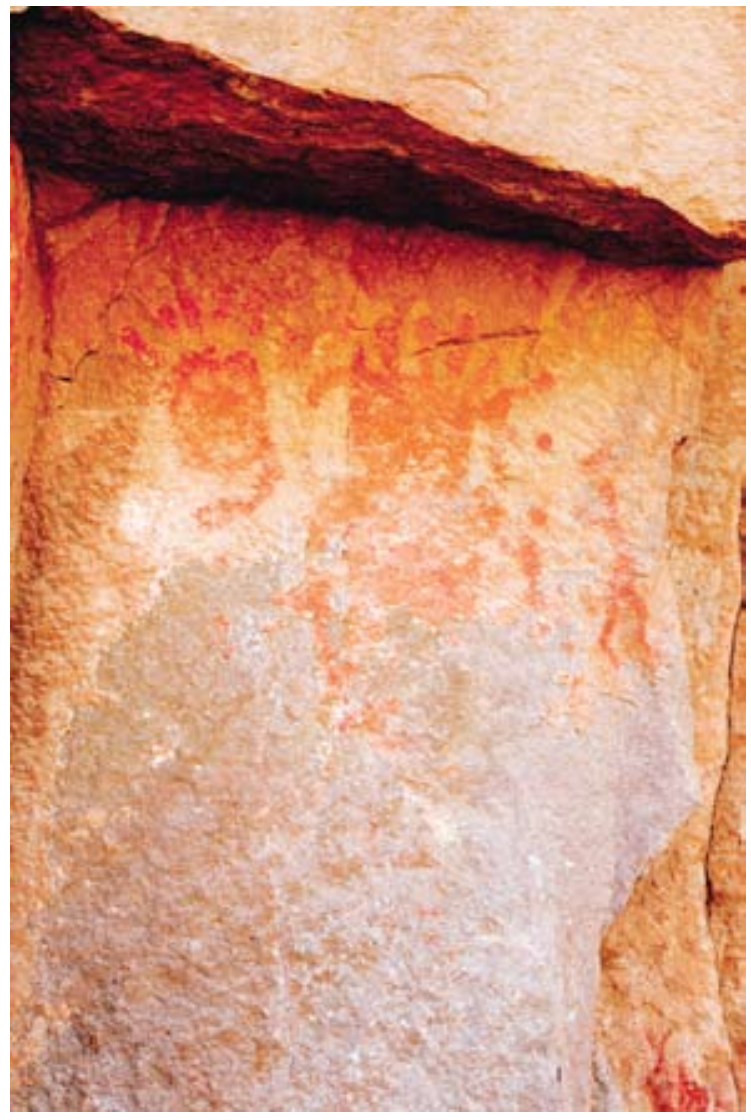

Figura 9. Pérdida de capa pictórica causada por eflorescencias salinas, Alto Loa (foto: A. Murillo, 2010).

Figure 9. Loss of painted layer due to saline efflorescence, Alto Loa (photo: A. Murillo, 2010).

generación de material particulado y su posterior depósito sobre las superficies. Adicionalmente, algunas de las cuadrículas se encuentran aún abiertas, lo que facilita procesos erosivos del suelo. De forma general, se registró un bajo porcentaje de intervenciones subactuales, situación atribuida nuevamente a la localización de los soportes. A la mayoría de los sitios se puede acceder solo escalando la escarpada superficie del talud, ya que en muy pocos emplazamientos existen senderos de acceso directos desde la cota del río. Sin embargo, la mayor cantidad de las intervenciones humanas se registraron en los aleros, donde un 35\% de los paneles presenta incisiones sobre las pinturas (fig. 11) y un $0,5 \%$, ahumado.

En los cinco tipos de soportes se registró similar porcentaje de alteraciones vinculadas a las oscilaciones térmicas, propias del sector. Debido a la altura, estas variaciones se registran de forma más intensa en intervalos diarios, con diferencia de hasta $30^{\circ}$ entre la mínima y la máxima (DGAC 2009). Esta situación provoca la expansión y la contracción de la roca y su consecuente fractura 


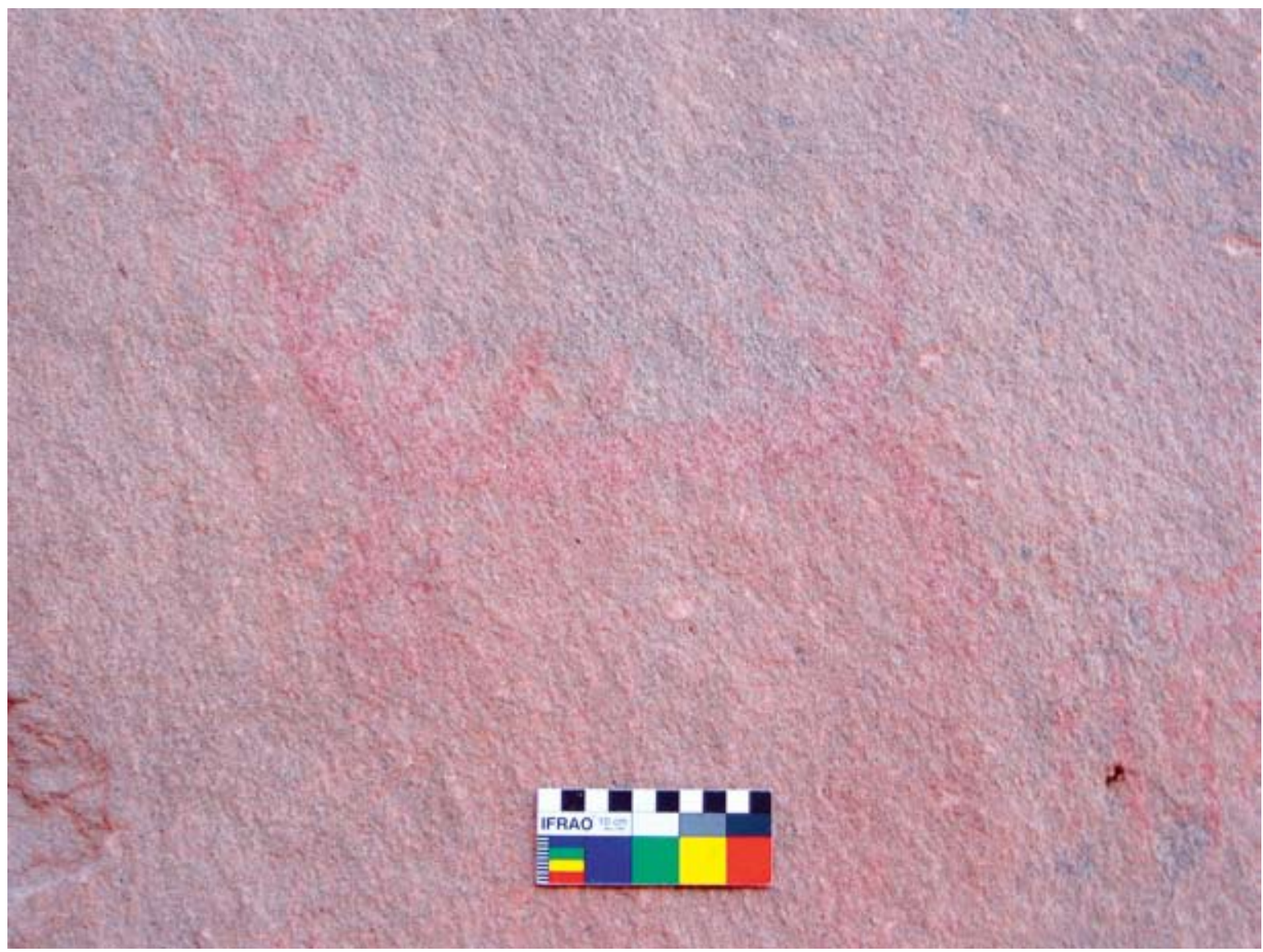

Figura 10. Depósito de sedimentos sobre la superficie de pictografía, Alto Loa (foto: I. Navarro, 2007). Figure 10. Sediment deposits on pictograph surface, Alto Loa (photo: I. Navarro, 2007).

(Wainwright 1995), la cual en este caso se manifiesta como grietas y exfoliación de los soportes.

\section{Ejecución de las representaciones}

Se consignó que en un $88 \%$ del total de los paneles se observan conjuntos tonales compuestos por matices rojos, un $2 \%$ presenta amarillos y un $2 \%$, verdes. En un $9 \%$ de los paneles se registran conjuntos de matices intermedios amarillo-rojo, mientras que matices neutros-blancos se relevan en un $24 \%$ de los soportes. Finalmente, un $4 \%$ de estos presentan pigmentos negros. En numerosos paneles existe más de un conjunto tonal, los que no siempre evidencian las mismas características estilísticas y grados de saturación de los matices. ${ }^{9}$

\section{Relación entre ejecución de las representaciones, factores e indicadores de alteración}

La relación más relevante entre los procesos de ejecución de las representaciones y los factores e indicadores de alteración registrados se establece al comparar el estado de conservación que presentan los diversos conjuntos tonales identificados. En el Gráfico 4 se representa la distribución de los indicadores de alteración según estos grupos.

En términos de impacto, el principal indicador de alteración registrado fue la pérdida de material pictórico, hecho que se evidencia como un desprendimiento laminar de la materia colorante desde los soportes y/o como la disgregación pulverulenta de la misma.

La pérdida de adhesión de la capa pictórica al sustrato es la alteración más importante en términos porcentuales, registrándose en un $40 \%$ del total de los paneles del sector. Un $94 \%$ de las representaciones efectuadas con pinturas blancas $(n=50)$ presentan desprendimientos, los que en varios casos se encuentran asociados a fisuras (fig. 12). Un 32\% de las de color rojo registró este cambio $(n=184)$. Esta relación se vincula con la variable consistencia de las mezclas pigmentarias. En un 100\% de los conjuntos tonales blancos la consistencia de la mezcla es pastosa presentando una menor adhesión al 


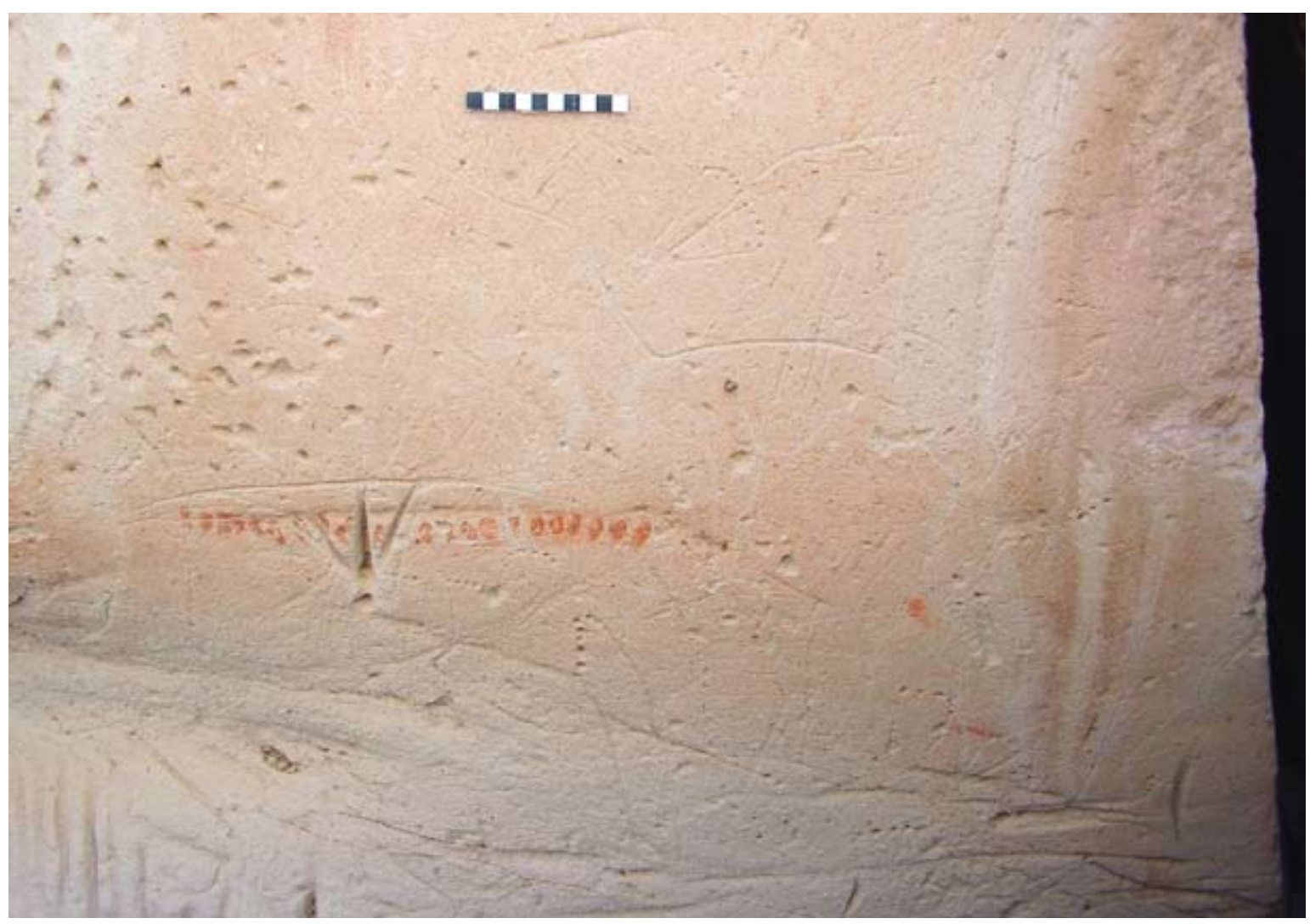

Figura 11. Sitio LS036. Detalles de incisiones sobre pintura, Alto Loa (foto: P. Casanova, 2007).

Figure 11. Site LSO36. Detail of incisions over paint, Alto Loa (photo: P. Casanova, 2007).

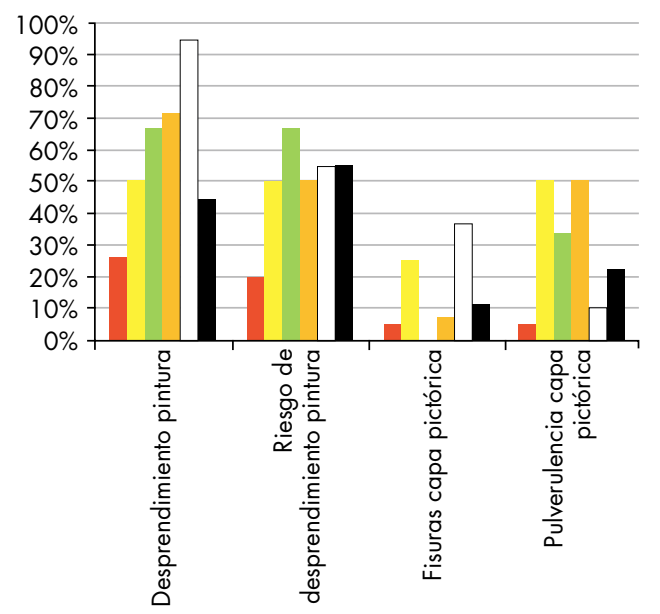

Rojo $(n=184 ; 88 \%)^{*} \quad$ Amarillo $(n=4 ; 2 \%)^{*} \quad$ Verde $(n=4 ; 2 \%)^{*}$

Amarillo-rojo $(\mathrm{n}=18 ; 9 \%)^{*} \square$ Blanco $(\mathrm{n}=50 ; 24 \%)^{*}$ Negro $(\mathrm{n}=9 ; 4 \%)^{*}$

${ }^{*}$ Cantidad de conjuntos tonales registrados $\mathrm{N}=269$

Gráfico 4. Distribución de los principales indicadores de alteración según conjuntos tonales registrados en 210 paneles con pintura rupestre, sector Alto Loa. Observación in situ con lupa portátil de 30X.

Graph 4. Distribution of main alteration indicators by color sets recorded on 210 rock art panels, Alto Loa Sector. In situ observation with portable $30 X$ magnifier. soporte. En el caso de los colores rojos, en un mismo porcentaje, se distingue una consistencia fluida y mayor incorporación a la roca.

La pérdida de cohesión de las partículas se manifiesta como pulverulencia o desintegración de la capa pictórica. Esta alteración se registró con mayor frecuencia en los tonos amarillos, amarillos-rojos y negros. En la figura 13 se observa una pintura polícroma y el diferente estado de conservación de las mezclas pigmentarias utilizadas.

Con respecto a los procedimientos de aplicación de las pinturas, se evidencia que las figuras ejecutadas con técnica mixta presentan diferentes grados de conservación entre sus trazos. En estos casos los motivos, en su mayoría de color rojo, son delimitados linealmente en su contorno, mientras que el espacio interior es abarcado de forma areal. De este modo, el perímetro presenta diversas capas de pintura superpuestas, lo cual deriva en su mayor espesor y mejor preservación (fig. 14). De forma general, cuando la aplicación de la pintura presenta superposición de capas aumenta la saturación del color, especialmente en las mezclas rojas, las cuales son más fluidas. Particularmente, en los estilos naturalistas (Taira y Milla) se observan zonas superpuestas, ya sea de trazos o motivos. En estos casos, 


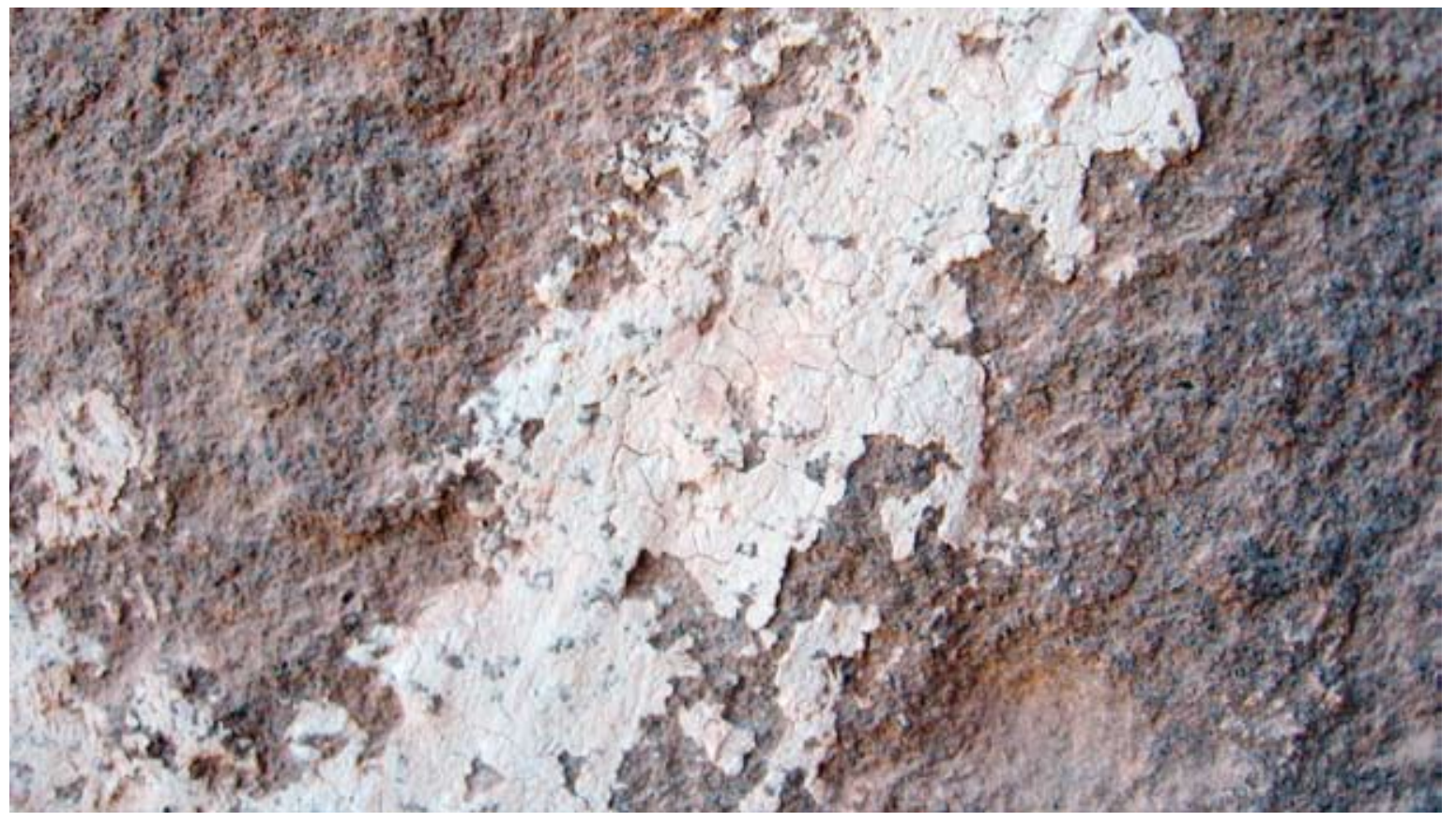

Figura 12. Sitio LS012. Detalle de microfisuras de mezcla pigmentaria blanca, Alto Loa (foto: P. Casanova, 2007).

Figure 12. Site LSO12. Detail of white pigment microfissures, Alto Loa (photo: P. Casanova, 2007).

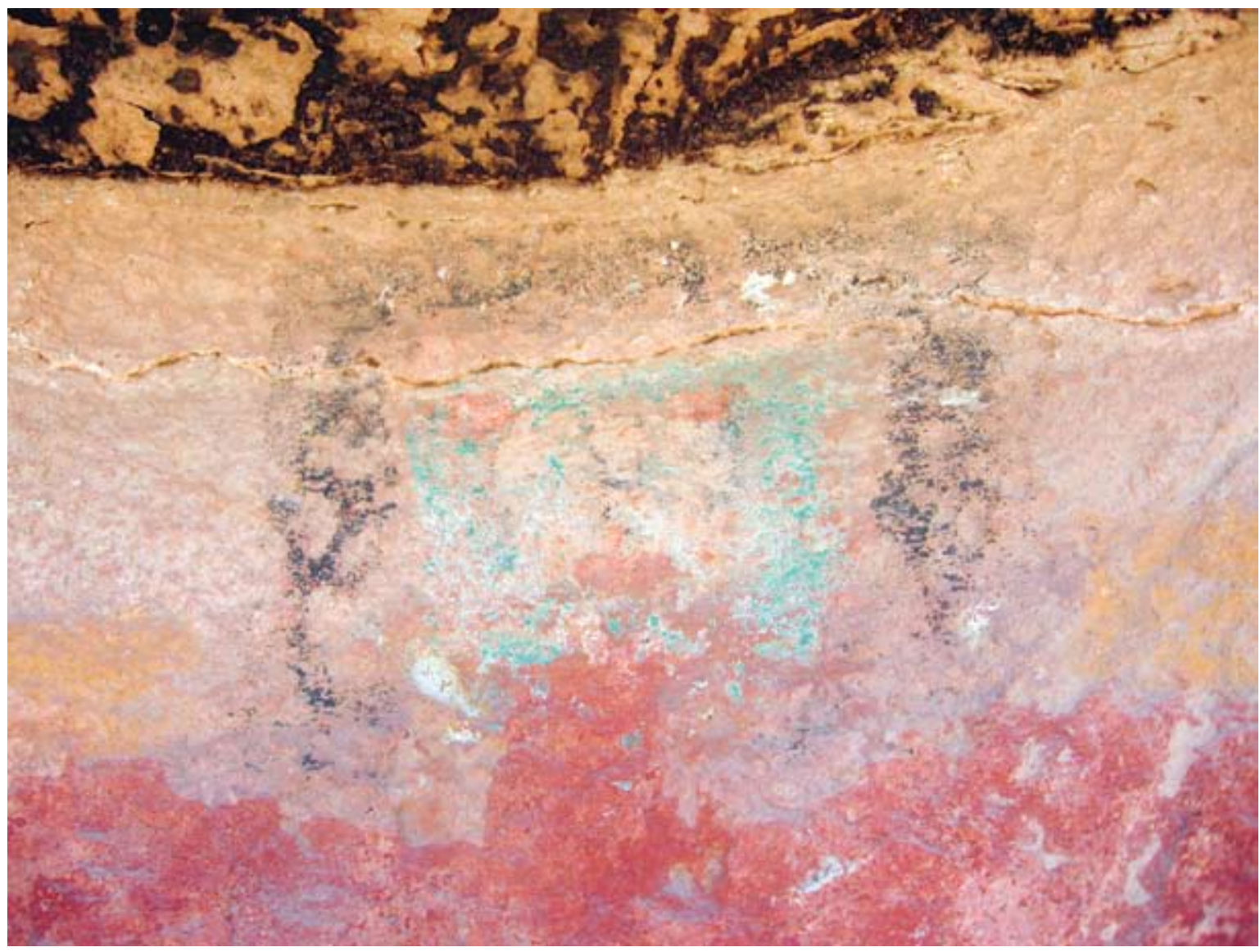

Figura 13. Conservación diferencial de diversos tonos (rojo, negro, verde, amarillo-rojo). Alero Santa Bárbara, Alto Loa (foto: P. Casanova, 2007). Figure 13. Differential conservation of colors (red, black, green, yellowish-orange). Santa Bárbara rock shelter, Alto Loa (photo: P. Casanova, 2007). 


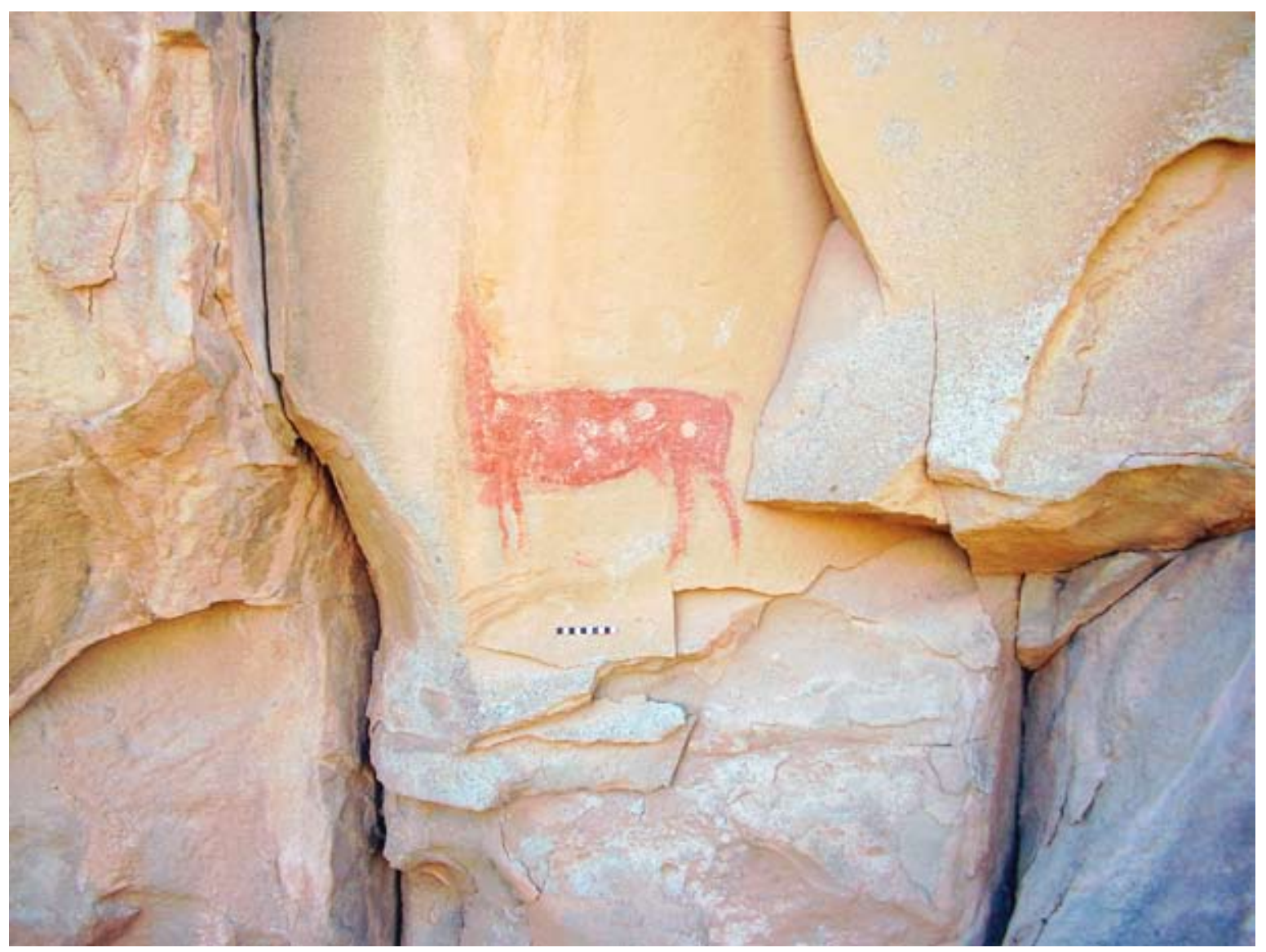

Figura 14. Pictografía zoomorfa realizada con técnica mixta (lineal y areal), Alto Loa (foto: P. Casanova, 2007).

Figure 14. Zoomorphic pictograph made with mixed technique (linear and areal), Alto Loa (photo: P. Casanova, 2007).

las sucesivas películas de color producen un efecto de mayor intensidad cromática.

\section{DISCUSIÓN}

Tras el análisis de los resultados obtenidos en esta evaluación se establecen las siguientes conclusiones y reflexiones.

Considerando el estado de integridad física de las pinturas rupestres, desde una perspectiva global, se plantea que estas se encuentran estables. Si bien la mayoría de las alteraciones registradas no son reversibles, en gran medida estas no presentan un grado de impacto que impida la apreciación holística de las figuras y, por tanto, su documentación e interpretación arqueológica. Según su localización, los sustratos utilizados como soporte presentan una mayor o menor exposición ante los factores externos presentes en el sector. Se estima que esta condición no es excluyente, es decir, las principales alteraciones registradas, salvo las incisiones, se observan en todos los tipos de soportes; lo que varía es su recurrencia e intensidad. Los cambios observados se han desarrollado paulatinamente a través de cientos de años y hoy se registra un equilibrio entre los recursos y el medio. Adicionalmente, se puede establecer que la dificultad para acceder a las representaciones es un factor positivo en cuanto la escasa incidencia de intervenciones vandálicas y presiones turísticas. Sin embargo, es interesante destacar que desde tiempos prehispánicos (Berenguer 1995, 1999; Cáceres \& Berenguer 1996; Horta 1996) hasta el día de hoy, algunos sitios mayormente protegidos son utilizados y reutilizados esporádicamente como refugio. Estos emplazamientos son los únicos que presentan indicadores exclusivos de alteración. La erosión, las incisiones y el ahumado de algunos emplazamientos son huellas de esta situación. Actualmente, pastores y pescadores de río dejan además rastro material de su paso (fig. 15).

A partir de esta primera evaluación general, se considera necesario incentivar nuevas acciones de conservación indirecta (Brunet et al. 1990), que profundicen en el 


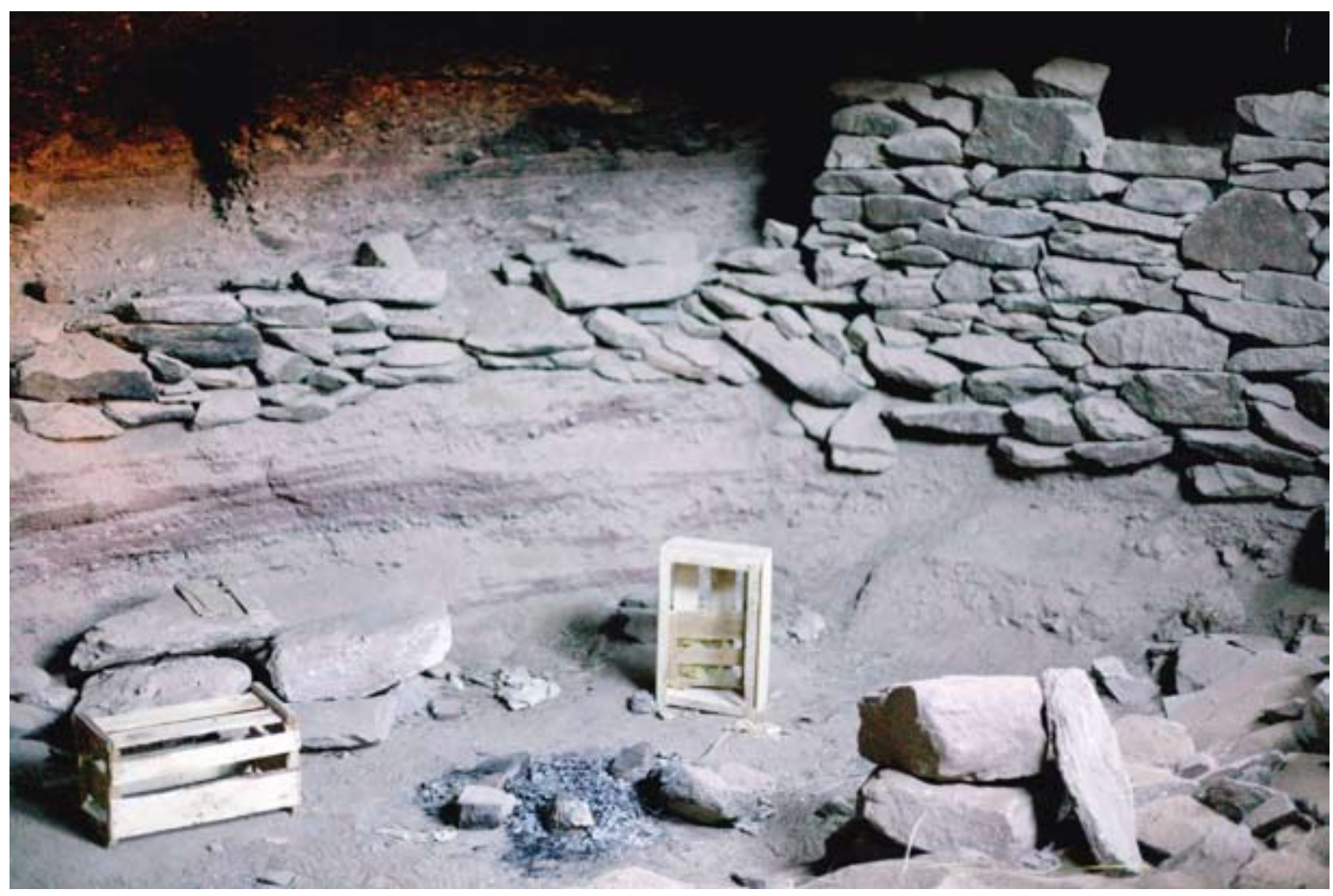

Figura 15. Reutilización actual de sitio Cueva La Damiana, Alto Loa (foto: A. Murillo, 2010)

Figure 15. Modern re-usage of Cueva La Damiana site, Alto Loa (photo: A. Murillo, 2010).

estudio de aspectos hídricos, geológicos y climáticos (Bednarik 1995, 2002) presentes en el sector y los sitios arqueológicos. Se requiere que equipos interdisciplinarios y especializados puedan establecer con mayor precisión la periodicidad de estos agentes y en algunos casos su acción interdependiente. Además, considerando las particularidades de cada uno de los emplazamientos, se requiere ahondar en el estudio del ambiente litológico (Rocchietti 2003) y la caracterización petrográfica de los soportes, incluyendo aspectos como la porosidad, la permeabilidad y la dureza de estos.

Una segunda conclusión se establece a partir de casos puntuales de estudio. Si bien el estado general de las pinturas es estable, se identifican dos puntos de vulnerabilidad y alto riesgo en la preservación de los recursos. El primero se relaciona con la pérdida de la configuración de algunas representaciones, causada por procesos de cristalización de sales. En estos casos, el impacto de los agentes de alteración es alto, en términos de intensidad y extensión de la superficie que abarcan. Son procesos activos e irreversibles. El segundo se vincula con la pérdida del material pictórico, ya sea a partir del desprendimiento laminar de la materia colorante desde los soportes o la disgregación y pulverización de la misma. Este tipo de cambio también es irreversible y se encuentra activo, conformando un riesgo inminente de pérdida de información arqueológica.

Es en este último punto donde se establece con mayor claridad la relación entre indicadores de alteración y los procesos de producción de las pinturas (Aschero 1988), particularmente, los involucrados en la preparación de las mezclas pigmentarias (Chalmin et al. 2003). Los factores intrínsecos propios de cada modo de hacer significan un resultado diferente en cuanto a la conservación de las representaciones registradas. Esta conclusión se obtiene al observar los patrones de alteración presentes en estas manifestaciones, especialmente comparando el alto grado de estabilidad de las representaciones ejecutadas con mezclas pigmentarias rojas y la presencia de nuevas alteraciones en los casos en que se amplía el repertorio cromático. Resultados de los primeros análisis elementales (SEM-EDX) elaborados durante el proyecto arqueológico identifican la presencia de óxido de hierro (hematita) en los pigmentos rojos (Sepúlveda 2008 Ms). A nivel mundial, el uso de estos compuestos ha sido consignado en numerosos sitios, destacándose en muchos casos la estabilidad de la hematita con respecto a diversos tipos de pigmentos (Bednarik 2002; Hradil 
et al. 2003; Morwood 2003; Argüello \& Martínez 2004; Wainwright \& Raudsepp 2008, entre otros). Por lo tanto, no es sorpresa que en el Alto Loa las representaciones ejecutadas con estos compuestos prevalezcan de mejor forma a través del tiempo. Considerando los diferentes períodos y estilos en que fueron realizadas las pinturas, y excluyendo los factores externos de alteración, se concluye que no existe una variación en el grado de preservación de las figuras que utilizaron mezclas pigmentarias rojas, y por ende, tampoco una modificación importante en su preparación. Sin embargo, la forma cómo es aplicada la mezcla, en especial la cantidad de capas superpuestas, incide en una mayor intensidad cromática de las figuras.

La incorporación de un nuevo repertorio cromático, sobre todo los verdes, blancos y negros, significa una mayor pérdida de los registros. Este cambio debería relacionarse con nuevas propiedades de la materia incorporada y/o la forma de producir las mezclas pigmentarias. Para ahondar en estas observaciones realizadas in situ se requiere de nuevos análisis científicos. ¿Se atribuyen al tamaño y la forma de las partículas las diferentes propiedades adhesivas de las mezclas a los soportes y/o la cohesión de las partículas de pigmento entre sî́? (Clarke 1976; Wainwright 1995; Morwood 2003); ies el uso de aglutinantes el elemento favorecedor de estas propiedades? (véase Petit \& Vallot 1991; Chalmin et al. 2003; Yacobaccio et al. 2008, entre otros).

Por otra parte, la presencia de estas alteraciones de alto riesgo nos lleva a reflexionar sobre la necesidad de intervenciones de tipo directa sobre las pinturas (Brunet 2006; Brunet et al. 1990). Si bien se han publicado diferentes métodos para aminorar el impacto del flujo superficial de agua sobre las paredes (Bednarik 1995; Brunet 1995), eliminar depósitos de sedimentos sobre las pinturas (Lambert 1989) y los problemas de sales vinculadas a la hidrología de los sitios (Bednarik 1995), en este contexto se cree que no es aconsejable aplicar acciones de este tipo, salvo el cierre de las cuadrículas que se encuentran abiertas y la protección de las pinturas en caso de nuevas excavaciones. Aunque existen razones técnicas e incluso presupuestarias para este planteamiento, se considera más relevante aún el hecho de que en el sector no existe una entidad administrativa responsable que decida y priorice sobre la conservación de los emplazamientos. Stanley Price (1995) plantea que son los administradores quienes, a partir del significado asignado a los sitios, deben tomar esta responsabilidad.

Ciertamente, gestiones administrativas se ven bastante lejanas a nuestra realidad, por lo que se propone en un comienzo definir al menos qué valores se identifican en los emplazamientos, explicitando su significado (Wainwright 1995; Australian Heritage Commission 2001). Un diálogo entre las partes interesadas en la preservación de este patrimonio (científicos, sector de turismo, comunidad, etcétera) es básico para establecer prioridades de conservación (Bellelli 2006) y diseñar estrategias para un uso sustentable de los recursos (Molinari et al. 2001).

Con respecto a este tema, en las últimas décadas se ha buscado desarrollar una mirada holística hacia los bienes patrimoniales, integrando aspectos culturales, sociales e incluso económicos. Desde este enfoque se plantean propuestas relacionadas con la gestión, la planificación y el manejo de los sitios arqueológicos (Lambert 1989; Marymor 2001; Australian Heritage Commission 2001; Morwood 2003, entre otros). Sin embargo, varios artículos exponen también las dificultades y las consecuencias de abrir al público estos recursos (Gale 1985; Gale \& Jacobs 1986; Bednarik 2003; Bellelli \& Podestá 2006, entre otros). Desde nuestra perspectiva, se considera indispensable definir un ente administrativo responsable antes de fomentar el turismo local. Considerando los enfoques planteados por diversos autores sobre patrimonio, uso y participación social (García Canclini 1999; Molinari et al. 2001; Caraballo 2006; Forero 2008), se estima que son las comunidades identificadas con el sector las que podrían adquirir este rol. De esta forma, la gestión cultural de los recursos (Ballard \& Tresserras 2001) se sustentaría sobre tres pilares: las instituciones públicas, la academia y la sociedad civil (Forero 2008), integrando los diversos valores que a este patrimonio le son atribuidos y compartiendo responsabilidades sobre su conservación.

En síntesis, en el sector del Alto Loa las pinturas rupestres se encuentran en un estado de conservación que presenta dos dimensiones. Por un lado, el carácter estable asignado a la mayoría de las pinturas radica en el equilibrio que estas han alcanzado con el medio, la localización de los paneles que permite su resguardo ante factores antrópicos y la buena preservación de las representaciones ejecutadas con pigmentos rojos (mezclas de hematita). Por otra parte, la incorporación posterior de diversos tonos, es decir, nuevas mezclas pigmentarias, deriva en una disminución significativa de la estabilidad de las representaciones, especialmente causada por la pérdida de adhesión y cohesión de las capas pictóricas. Ante las presiones del turismo informal, se plantea que no es necesario esperar el aumento del flujo de visitantes, ni que ocurran pérdidas irreparables para tomar acciones precautorias. Tampoco es pertinente impulsar el desarrollo turístico en la zona si no existe un ente administrativo responsable de velar por la integridad de los recursos. 
Si bien esta evaluación es preliminar, el trabajo realizado plantea una mirada a largo plazo, que profundice en los aspectos naturales del entorno rupestre, su administración y que además trascienda hacia la dimensión social de los bienes.

RECONOCIMIENTOS Proyecto FondeCyT No 1070083, Francisco Gallardo, Iván Maureira, Isabel Navarro, Marcela Sepúlveda, Soledad Fernández, Daniela Valenzuela, Manuel Alarcón, Verónica Silva, Paulina Corrales, Augusto Murillo, familia Galleguillos, Paula Ugalde, Arlene Muñoz, María José Galleguillos, Carla Loayza.

\section{NOTAS}

${ }^{1}$ Este diagnóstico preliminar del estado de conservación de las pinturas se realiza en el marco del proyecto FONDECYT "Pinturas rupestres, estilos tecnológicos y flujos de información visual en la región atacameña y áreas vecinas".

${ }^{2}$ En algunos estudios arqueológicos esta condición se aborda de manera tangencial (ver Horta 1996, 2000; Berenguer 1995).

${ }^{3}$ A diferencia del término "Loa Superior", el cual incluye no solo el curso superior de este río, sino también la totalidad de la hoya del río Salado (Berenguer et al. 2005).

${ }^{4}$ Berenguer (2004) precisa que La Isla, más que un estilo, se comporta como una iconografía rupestre.

${ }^{5}$ Por indicador de alteración se entiende aquellas manifestaciones de cambio apreciables en las propiedades fisicomecánicas de los recursos (Martín 1990). En este caso, se realizó una apreciación cualitativa de aquellas modificaciones observables a simple vista. Por factor de alteración se entiende "aquellas circunstancias, hechos o influencias que contribuyen a un determinado grado de alteración, creando las condiciones para que tenga lugar un cambio o bien influyan sobre los mecanismos que provoquen los mismos (Martín 1990: 42). Se consignaron las variables extrínsecas (naturales, antrópicas, biológicas) e intrínsecas.

${ }^{6}$ En este estudio nos referiremos al término pigmento en su acepción general, es decir, como una sustancia coloreada que confiere su color a otro material, el cual puede ser de origen mineral u orgánico, su naturaleza arcillosa o no arcillosa (óxidos de hierro) (Hradil et al. 2003) y que es utilizado sistemáticamente en la composición y la confección de pinturas (mezclas pigmentarias) (Petit \& Vallot 1991).

${ }^{7}$ Esta clasificación debe complementarse con un mayor análisis de los sustratos, incluyendo su composición mineralógica y textura.

8 Promedio calculado para períodos inferiores a 30 años.

${ }^{9}$ La mayoría de las representaciones fueron plasmadas sobre soportes que presentan cualidades muy similares en cuanto a color y textura, por lo tanto, las diferencias cromáticas en términos de saturación del color no han sido atribuidas en primera instancia a estos aspectos.

\section{REFERENCIAS}

Alonso, J.; M. E. Rosa, J. Ordaz \& P. Vázquez, 2006. Análisis del deterioro en los materiales pétreos de edificación. Revista Electrónica RECOPAR 3, 2005-2008 [online] pp. 23-32<http:// www.recopar.com/pdf/RECOPAR-N3.pdf> ISSN 1886-2497 [Citado 25-10-09].

Argüello, P. \& D. Martínez, 2004. Procesos tafonómicos en el arte rupestre: un caso de conservación diferencial de pinturas en el altiplano cundiboyacense, Colombia [online] <http://rupestreweb.tripod.com/sutatausa.html> [Citado 04-11-09].
Aschero, C., 1988. Pinturas rupestres, actividades y recursos naturales: un encuadre arqueológico. En Arqueología contemporánea argentina: Actualidad y perspectivas, H. Yacobaccio, Ed., pp. 109-145. Buenos Aires: Ediciones Búsqueda.

Australian Heritage Commission, 2001. Successful Tourism at Heritage Places: A guide for tourism operators, heritage managers and communities. Australian Heritage Commission and CRC for Sustainable Tourism, Canberra [online] pp. 1-6 <http://www. environment.gov.au/heritage/ahc/publications/commission/ books/pubs/successful-tourism.pdf> [Citado 09-07-10].

Ávalos, H. \& B. Ladrón de Guevara, 2000. El patrimonio cultural como bien de consumo: el caso Petorca. Conserva 4: 87-114. Santiago: DIBAM.

Ballard, J. \& J. Tresserras, 2001. Gestión del patrimonio cultural. Barcelona: Ariel Editores.

BEDNARIK, R., 1995. Conservación del arte rupestre en Australia. Administración y conservación de sitios de arte rupestre: Contribuciones al estudio del arte rupestre sudamericano 4: 9-21. La Paz: Sociedad de Investigación del Arte Rupestre en Bolivia.

2002. Arte rupestre, tafonomía y epistemología [online] <http:// mc2.vicnet.net.au/home/auraesp/web/epistemologia.html> [Citado 26-05-10].

- 2003. Natural deterioration of rock art. Rock art conservation [online] <http://mc2.vicnet.net.au/home/conserv/web/natural. html> [Citado 07-04-10].

Bellelli, C., 2006. Arqueología y patrimonio: Una historia de usos y abusos en el valle medio del río Chubut, Patagonia argentina. En Tramas en la piedra: Producción y usos del arte rupestre, D. Fiore \& M. M. Podestá, Eds., pp. 251-262. Buenos Aires: Sociedad Argentina de Antropología y Asociación Amigos del INAPL.

Bellelli, C.; M. Carballido, P. Fernández, M. Paniquelli, M. M. Podestá, V. Scheinsohn \& C. Sierra, 1999. La Comarca Andina del Paralelo $42^{\circ}$ : Protección y preservación del arte rupestre. En Actas del II Congreso de Historia Social y Política de la Patagonia Argentino-Chilena, pp. 105-113. Rawson: Secretaría General de la Gobernación de la Provincia del Chubut.

Belleldi, C.; V. Scheinsohn, M. M. Podestá, M. Carballido, P. Fernández \& S. CARACOTCHE, 2005. Arte rupestre y turismo: Comarca Andina del Paralelo $42^{\circ}$, Argentina. Estudios y perspectivas en turismo 14 [online] pp. 22-50< http://www.scielo.org.ar/pdf/eypt/v14n1/ v14n1a02.pdf> ISSN 1851-1732 [Citado 06-08-2010].

Bellelli, C. \& M. M. Podestá, 2006. Integración de sitios con arte rupestre a emprendimientos ecoturísticos en la Patagonia. El caso del valle del río Manso Inferior. En Tramas en la piedra: Producción y usos del arte rupestre, D. Fiore \& M. M. Podestá, Eds., pp. 238-250. Buenos Aires: Sociedad Argentina de Antropología y Asociación Amigos del INAPL.

Berenguer, J., 1995. El arte rupestre de Taira dentro de los problemas de la arqueología atacameña. Chungara 27 (1): 7-43.

- 1996. Identificación de camélidos en el arte rupestre de Taira: ¿Animales silvestres o domésticos? Chungara 28 (1-2): 85-114.

- 1999. El evanescente lenguaje del arte rupestre en los Andes atacameños. En Arte rupestre en los Andes de Capricornio, J. Berenguer \& F. Gallardo, Eds., pp. 9-56. Santiago: Museo Chileno de Arte Precolombino.

- 2002. Tráfico de caravanas, interacción interregional y cambio cultural en la prehistoria tardía del desierto de Atacama. PhD Dissertation, University of Illinois at Urbana-Champaign.

2004a. Cinco milenios de arte rupestre en los Andes atacameños: Imágenes para lo humano, imágenes para lo divino. Boletín del Museo Chileno de Arte Precolombino 9: 75-108.

_ 2004b. Caravanas, interacción y cambio en el desierto de Atacama. Santiago: Sirawi Ediciones.

Berenguer, J.; V. Castro, C. Aldunate, C. Sinclaire \& L. Cornejo, 1985. Secuencia del arte rupestre en el Alto Loa: Una hipótesis de trabajo. En Estudios en Arte Rupestre, C. Aldunate, J. Berenguer \& V. Castro, Eds., pp. 87-108. Santiago: Museo Chileno de Arte Precolombino. 
Berenguer, J. \& J. L. Martínez, 1986. El río Loa, el arte rupestre de Taira y el mito de Yakana. Boletín del Museo Chileno de Arte Precolombino 1: 79-99.

Berenguer, J.; I. Cáceres, C. Sanhueza \& P. Hernández, 2005. El Qhapaqñan en el Alto Loa, norte de Chile: Un estudio micro y macromorfológico. Estudios Atacameños 29: 7-39.

BRunEt, J., 1995. Presentación de la conservación del arte prehistórico en Francia. Administración y conservación de sitios de arte rupestre: Contribuciones al estudio del arte rupestre sudamericano 4: 29-45. La Paz: Sociedad de Investigación del Arte Rupestre en Bolivia.

- 2006. La conservation des grottes et des abris préhistoriques. Monumental. Dossier Grottes ornées, semestriel 2: 22-29, Paris.

Brunet, J.; P. Vidal \& J. Vouvé, 1985. Conservation de l'art rupestre: deux études, glossaire illustré. Paris: unEsCo.

Brunet, J.; I. Dangas, P. Vidal \& J. Vouvé, 1990. La conservation de l'art des cavernes et des abris. Paris: Section Française de l'Institut International de Conservation.

Brunet, J. \& J. VouvÉ, 1996. La conservation des grottes ornées, Collection Conservation du Patrimoine. Paris: CNRS Editions.

Brunet. J.; A. Brunet, I. Dangas, E. Guillamet \& P. Vidal, 1996. Altérations, dégradations, traitements. En La conservation des grottes ornées, J. Brunet \& J. Vouvé, Eds., pp. 171-217. Paris: CNRS Editions.

Cáceres, I. \& J. Berenguer, 1996. El caserío de Santa Bárbara 41: Su relación con la wak'a de Taira, Alto Loa. Chungara 28 (1-2): 381-393.

Caraballo, C., 2006. El patrimonio cultural: ¿Capital social o capitalización de los bienes? En Actas del VIII Congreso Internacional de Rehabilitación del Patrimonio Arquitectónico y Edificación, S. Sullivan, C. Caraballo \& E. Carvajal, Eds., pp. 12-25. Buenos Aires: Centro Internacional para la Conservación el Patrimonio.

Chalmin, E.; M. Menu \& C. Vignaud, 2003. Analysis of rock art painting and technology of Palaeolithic painters. Measurement Science and Technology 14: 1590-1597.

Clarke, J., 1976. Two aboriginal rock art pigments from Western Australia: their properties, use and durability. Studies in Conservation 21 (3): 134-142. London: IIC.

DGAC 2009. Dirección Meteorológica de Chile. Climas de Chile Región Antofagasta [online] <http://www.meteochile.cl/climas/ climas_segunda_region. $\mathrm{html}{ }^{*} \mathrm{C}>$ [Citado 25-10-09].

Forero, E., 2008. Sustentabilidad, paisaje cultural, arqueología y turismo: bases para la gestión de proyectos, participación, conservación del patrimonio socionatural. International Journal of South American Archaeology 2, 2007-2008 [online] pp.19-32 $<$ http://www.ijsa.syllabapress.com/issues/articles/ijsa00010. html> ISSN 2011-0626 [Citado 15-10-09].

FORT, R., 1996. El agua en los materiales pétreos: comportamiento hídrico. En Degradación y conservación del patrimonio arquitectónico, F. Mingarro, Ed., pp. 237-248. Madrid: Editorial Complutense.

Gale, F., 1985. Monitoring visitor behaviour at rock art sites. Rock Art Research 2: 112-18. Melbourne: AURA.

GALE, F. \& J. JACOBS, 1986. Identifying high-risk visitors at aboriginal art sites in Australia. Rock Art Research 3 (1): 3-19. Melbourne: AURA.

Gallardo, F., 2007 Ms. Pinturas rupestres, estilos tecnológicos y flujos de información visual en la región atacameña y áreas vecinas. Proyecto FONDECYT No 1070083.

García Canclini, N., 1999. Los usos sociales del patrimonio cultural. En Patrimonio etnológico: Nuevas perspectivas de estudio, E. Aguilar, Ed., pp. 16-33. Granada: Consejería de Cultura de la Junta de Andalucía.

Gradín, J. C., 1978. Algunos aspectos del análisis de las manifestaciones rupestres. Revista del Museo Provincial I (1): 120-133, Neuquén.

HeRnÁNDEZ Llosas, M. I., 1985. Diseño de una guía para el relevamiento y clasificación de datos de sitios arqueológicos con arte rupestre. En Estudios en arte rupestre, C. Aldunate, J. Berenguer \& V. Castro, Eds., pp. 25-36. Santiago: Museo Chileno de Arte Precolombino.

HorTa, H., 1996. Taira: Definición estilística e implicancias iconográficas de su arte rupestre. Chungara 28 (1-2): 395-417.

— 2000. El arte de Taira. Definición estilística e iconográfica. Revista de Teoría del Arte 2. Santiago: Departamento de Teoría de las Artes, Facultad de Artes Universidad de Chile.

— 2001. Sectorización de estilos en el arte rupestre del Loa, norte de Chile. En Actas de Segundas Jornadas de Arqueología, J. Berenguer, L. Cornejo, C. Sinclaire \& F. Gallardo, Eds., pp. 85108. Santiago: Museo Chileno de Arte Precolombino.

Hradil, D.; T. Grygar, J. Hradilova \& P. Bezdicka, 2003. Clay and iron oxide pigments in the history of painting. Applied Clay Science 22 (5) [online] pp. 223-236. <http://www.iic.cas.cz/ grygar/ ACS-earths.pdf> [Citado 10-06-10]

Icomos, 1999. Carta Internacional sobre Turismo Cultural. La gestión del turismo en los sitios con patrimonio significativo. Adoptada por Icomos en la $12^{\mathrm{a}}$ Asamblea General, México [online] <http:// www.international.icomos.org/charters/tourism_sp.htm> [Citado 25-06-10].

Ladrón de Guevara, B., 2004. Problemas de conservación de los sitios de Combarbalá: primeros diagnósticos. Revista Werken 5: 109-113, Santiago.

LAMBERT, D., 1989. Conserving Australian Rock Art: A Manual for Site Managers, G. Ward, Ed. Canberra: Aboriginal Studies Press.

Martín, A., 1990. Ensayos y experiencias de alteración en la conservación de obras de piedra de interés histórico artístico. Madrid: Ediciones Centro de Estudios Ramón Areces.

MARYMOR, L., 2001. ARARA Guidelines for Managers of Rock Art Sites on Public Lands: Public Access, American Rock Art Research Association and Protection Committee [online] <http://www. arara.org/Guidelines_Managers_Public_Lands.pdf> [Citado 26-08-09].

Meléndez, B. \& J. M. Fuster, 1998. Geología. Madrid: Paraninfo Editores.

Molinari, R.; L. Ferraro, H. Paradela, A. Castaño \& S. Caracotche, 2001. Odisea del manejo: Conservación del patrimonio arqueológico y perspectiva holística. II Congreso Virtual de Antropología y Arqueología. NAYA [online] <http://www. naya.org.ar/congreso2000/ponencias/Roberto_Molinari2.htm> [Citado 08-05-10].

Morwood, M. J., 2003. Visions from the Past. The archaeology of Australian Aboriginal Art. St. Leonards, N. S. W.: Allen \& Unwin Editores.

Munsell@ Color Company -X-Rite, 2000. Munsell Soil Color Chart (Year 2000 Revised Washable Edition), Michigan.

NaRBona, J., 2009. Boletín información pluviométrica, estado de embalses y aguas subterráneas 376, agosto. Dirección General de Aguas, Ministerio de Obras Públicas, Chile [online] <http:// www.dga.cl/index.php?option $=$ content\&task $=$ category\&sectio nid=16\&id=43\&Itemid=169> [Citado 25-09-09].

Niemeyer, H., 1967. Un nuevo sitio de arte rupestre en Taira (Río Loa, Prov. Antofagasta, Chile). Revista Universitaria 52: 159164, Santiago.

OMT, 1999. Código ético mundial para el turismo. Adoptado por la resolución A/RES/406(XIII) de la decimotercera Asamblea General de la Organización Mundial del Turismo, Santiago.

Petit J. \& H. Vallot, 1991. Glossaire des peintures et vernis. Des substances naturelles et des matériaux synthétiques. Champs Sur Marne: Section Française de l'Institut International de Conservation-Association des Restaurateurs d'Art et d'Archéologie de formation universitaire-Institute Français de Restauration des CEuvres d'Art.

Podestá, M. M.; C. Bellelli, P. Fernández, M. Carballido \& M. Paniquelli, 2000. Arte rupestre en la Comarca Andina del Paralelo $42^{\circ}$ : un caso de análisis regional para el manejo de recursos culturales. En Arte en las rocas: Arte Rupestre, menhires y piedras de colores en Argentina, M. M. Podestá \& M. Hoyos, Eds., pp. 175-201. Buenos Aires: Sociedad Argentina de Antropología. 
Rocchietti, A. M., 2003. Sistematización de la documentación del ambiente rupestre. Rupestre/web [online] <http://rupestreweb. tripod.com/ambiente.html> [Citado 20-10-09].

Rolandi, D.; M. M. Podestá, M. Sánchez \& A. Re, 2002. Procesos de deterioro y diagnóstico del grado de preservación de sitios con pinturas rupestres: el caso de Las Juntas (Guachipas, Provincia de Salta). En Documentación y registro del arte rupestre: Contribución al estudio del arte sudamericano 6: 94-108. La Paz: Sociedad de Investigación del Arte Rupestre en Bolivia.

SepúlvedA, M., 2004. Esquemas visuales y emplazamiento de las representaciones rupestres de camélidos del Loa Superior en tiempos incaicos: ¿Una nueva estrategia de incorporación de este territorio al Tawantinsuyu? Chungara 36 (2): 439-451.

2008 Ms. Tecnología de las pinturas rupestres del Loa Superior, norte de Chile. Primeros resultados de análisis físico-químicos de los compuestos inorgánicos. Proyecto FondecyT 1070083.

Soleilhavoup, F., 1985. Les paysages de l'art rupestre de plein air: vers une normalisation des méthodes d'étude et de conservation. Rock Art Research 2: 119-139. Melbourne: AURA

STANLEy Price, N., 1995. Introducción. Administración y conservación de sitios de arte rupestre. Contribuciones al estudio del arte rupestre sudamericano 4: 7-8. La Paz: Sociedad de Investigación del Arte Rupestre en Bolivia.
UnEsCo, 2006. Tourism, Culture and Sustainable Development [online] $<$ http://unesdoc.unesco.org/images/0014/001475/147578e. pdf $>$ [Citado 14-05-10].

VILCHES, F., 2005. Espacio celeste y terrestre en el arte rupestre de Taira. Boletín del Museo Chileno de Arte Precolombino 10 (1): 9-34.

WAinwright, I. N. M., 1995. Conservación y registro de pinturas rupestres y petroglifos en Canadá. Administración y conservación de sitios de arte rupestre: Contribuciones al estudio del arte rupestre sudamericano 4: 52-81. La Paz: Sociedad de Investigación del Arte Rupestre en Bolivia.

WAinwRight, I. N. M. \& M. RAudSEPP, 2008. Identificación de pigmentos de pinturas rupestres de Paja Colorada, Prov. Vallegrande, Depto. de Santa Cruz. Boletín Sociedad de Investigación del Arte Rupestre de Bolivia 22: 41-45, La Paz.

Watchman, A., 2002. The impacts of dust in the conservation and management of Aboriginal rock paintings in northern Australia. En L'art avant l'bistoire: La conservation de l'art prébistorique. 10èmes journées d'études de la Section Française de l'Institut International de Conservation, R. Hocquette, Ed., pp. 25-34. Paris: SFICC.

Yacobaccio, H. D.; M. P. Catá, P. Solá \& M. S. Alonso, 2008. Estudio arqueológico y fisicoquímico de pinturas rupestres en Hornillos 2 (Puna de Jujuy). Estudios Atacameños 36: 5-28. 